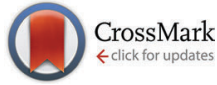

Cite this: Chem. Commun., 2014, 50, 12434

Received 9th June 2014 Accepted 11th July 2014

DOI: $10.1039 / c 4 c c 04381 b$

www.rsc.org/chemcomm

\section{Recent advances in the catalytic asymmetric 1,3-dipolar cycloaddition of azomethine ylides}

\author{
Javier Adrio* and Juan C. Carretero* \\ Catalytic asymmetric 1,3-dipolar cycloadditions of azomethine ylides have turned out to be one of the \\ most efficient methods for the preparation of enantioenriched pyrrolidines. The past decade has \\ witnessed the development of a bunch of well-defined catalytic systems capable of affording excellent \\ diastereo and enantioselectivities. Recently, a great effort has been focused on expanding the scope of \\ the cycloaddition with regard to both reaction partners. In this review, we will discuss important advances \\ that have been reported in this area since 2011.
}

\section{Introduction}

The intrinsic efficiency and atom economy of the cycloaddition reactions (several bonds and stereocenters are formed in a single reaction) have determined its paramount importance in modern organic synthesis. ${ }^{1}$ On the other hand, industry demands more selective and sustainable procedures for the preparation of structurally complex enantiomerically enriched compounds. ${ }^{2}$ Consequently, a great effort has been devoted to the development of efficient catalytic asymmetric cycloaddition procedures. In this context, the 1,3-dipolar cycloadditions play a crucial role in the enantioselective preparation of five membered heterocycles. ${ }^{3}$ In particular, the catalytic asymmetric 1,3-dipolar cycloaddition of azomethine yides with activated olefins has become one of the most useful and well-established methods for the preparation of enantioenriched substituted pyrrolidines, a structural moiety widely present in a myriad of natural products and biologically active compounds. ${ }^{4}$

In 2002, inspired by the previous work by Grigg and co-workers ${ }^{5}$ using stoichiometric amounts of $\mathrm{CoCl}_{2}$ as metal salt and an ephedrine derivative as a chiral ligand, the research groups of Zhang $^{6}$ (using a chiral Ag complex) and Jørgensen ${ }^{7}$ (Zn chiral Lewis acid), independently, reported the first examples of the catalytic enantioselective 1,3-dipolar cycloaddition of azomethine ylides derived from iminoesters and activated olefins.

The great effectiveness of this process relays on the formation of a metalated azomethine ylide by the coordination of the nitrogen and oxygen atoms to the metal. This rigid five membered metallocycle makes easier the deprotonation of the enolizable $\mathrm{C}-\alpha$ position and the asymmetric induction from the

Departamento de Química Orgánica, Facultad de Ciencias, Universidad Autónoma de Madrid, Cantoblanco, 28049 Madrid, Spain.

E-mail: javier.adrio@uam.es, juancarlos.carretero@uam.es; Fax: +34 914973966

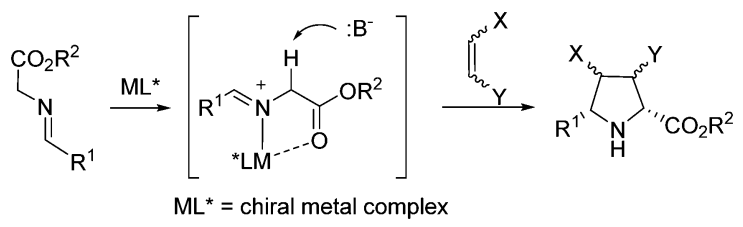

Scheme 1 Classical catalytic asymmetric 1,3-dipolar cycloaddition using $\alpha$-iminoesters as dipole precursors.

chiral ligand (Scheme 1). The intrinsic limitation of this strategy is that it always provides pyrrolidines with a C-2 carboxylate ester substitution.

During the next decade a great effort has been focused on the development of more general and efficient catalytic systems, as well as the improvement of the structural scope of this asymmetric process. As a result, an assortment of chiral metal complex catalysts has been described. Although the most commonly used metals are $\mathrm{Cu}$ and $\mathrm{Ag}, \mathrm{Zn}, \mathrm{Ni}, \mathrm{Ca}$, and $\mathrm{Au}$ chiral complexes have also been effectively employed. A key issue in the success of the process is the use of an efficient and stereodiscriminating chiral ligand to control the diastereo and enantioselectivity of the cycloaddition. In this regard, a great variety of structurally diverse ligands (mainly based on P,N; P,P; or P,S coordination modes) have been developed, among them Ugi amine-derived ligand 1, Fesulphos 2, Biphamphos $\mathbf{3}$ and Segphos $\mathbf{4}$ have emerged as examples of privileged ligands for this cycloaddition, affording excellent results for a wide range of substrates (Fig. 1).

Additionally, in the last few years organocatalysis has also emerged as an attractive alternative to the metal catalyzed procedures. The limitations found in the first organocatalyzed protocols, such as the need for deactivated azomethine ylide precursors or the required high catalyst loading, have been partially overcome with the discovery of more efficient organocatalysts. 

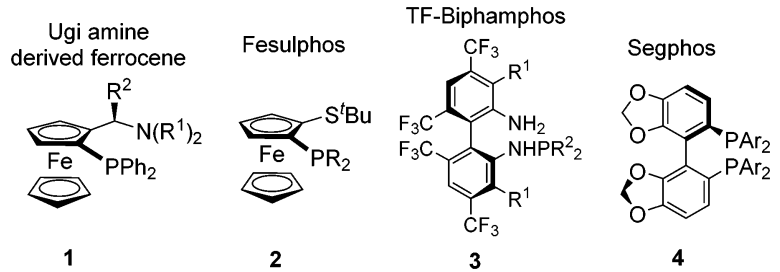

Fig. 1 Relevant privileged ligands in the 1,3-dipolar cycloaddition of azomethine ylides.

Remarkably, in the last few years the structural scope of this asymmetric cycloaddition has been greatly improved with the incorporation of novel types of dipolarophiles (different to the standard acrylates and related $\alpha, \beta$-unsaturated esters), and dipole precursors (e.g. $\alpha$-iminoamides, $\alpha$-iminophosphonates, $\alpha$-iminonitriles or $\alpha$-silylimines), as well as the first examples of higher order cycloadditions of azomethine ylides $(e . g .[3+3]$ and [6+3] cycloadditions).

In 2011, we published a review on the recent developments in this area. ${ }^{8}$ The present feature article focuses on the contributions achieved from 2011 to April 2014 with special emphasis on the results involving novel dipolarophiles and azomethine ylide partners. Finally, we also present the recent developments involving organocatalysis.

\section{New dipolarophiles for metal catalyzed cycloadditions}

The development of increasingly reactive catalytic systems for this cycloaddition has paved the way for the use of less reactive dipolarophiles. Therefore, the range of $\alpha, \beta$-unsaturated carboxylic acid derivatives has evolved from simple monoactivated (such as acrylates, enones, nitroalkenes and vinyl sulfones) or symmetrically double activated dipolarophiles (such as maleates, fumarates, maleimides, and fumaronitriles) to more varied and challenging alkenes as summarized next.

\subsection{Sterically demanding $\alpha, \beta$-unsaturated carbonyl derivatives}

In comparison with standard $\alpha, \beta$-unsaturated dipolarophiles, $\alpha$-substituted substrates, which lead to the formation of pyrrolidines bearing a quaternary stereocenter at C-4, have been much less explored. In fact, until recently, the sort of such dipolarophiles was basically restricted to the use of alkylidene malonates, ${ }^{9}$ methacrylate ${ }^{10}$ and methacrolein. ${ }^{11}$

In 2011 Wang and co-workers expanded the scope of the cycloaddition to hydroxymethyl methyl acrylates, easily available by the Morita-Baylis-Hillman reaction. ${ }^{12}$ Under optimized conditions $\left(3 \mathrm{~mol} \%\right.$ of $\mathrm{Cu}\left(\mathrm{CH}_{3} \mathrm{CN}\right)_{4} \mathrm{BF}_{4} / \mathrm{TF}-\mathrm{Biphamphos} 3 \mathrm{a}$, $15 \mathrm{~mol} \% \mathrm{Et}_{3} \mathrm{~N}$ in $\mathrm{CH}_{2} \mathrm{Cl}_{2}$ at $-20{ }^{\circ} \mathrm{C}$ ) a variety of pyrrolidines bearing a quaternary stereocenter were obtained with excellent diastereoselectivity and enantioselectivity (up to $97 \%$ ee). Unfortunately, the cycloaddition of the racemic alcohol $\left(\mathrm{R}^{2} \neq \mathrm{H}\right)$ led to two diastereomers 5 in a 1:1 ratio, with the kinetic resolution of the mixture not being observed (Scheme 2).
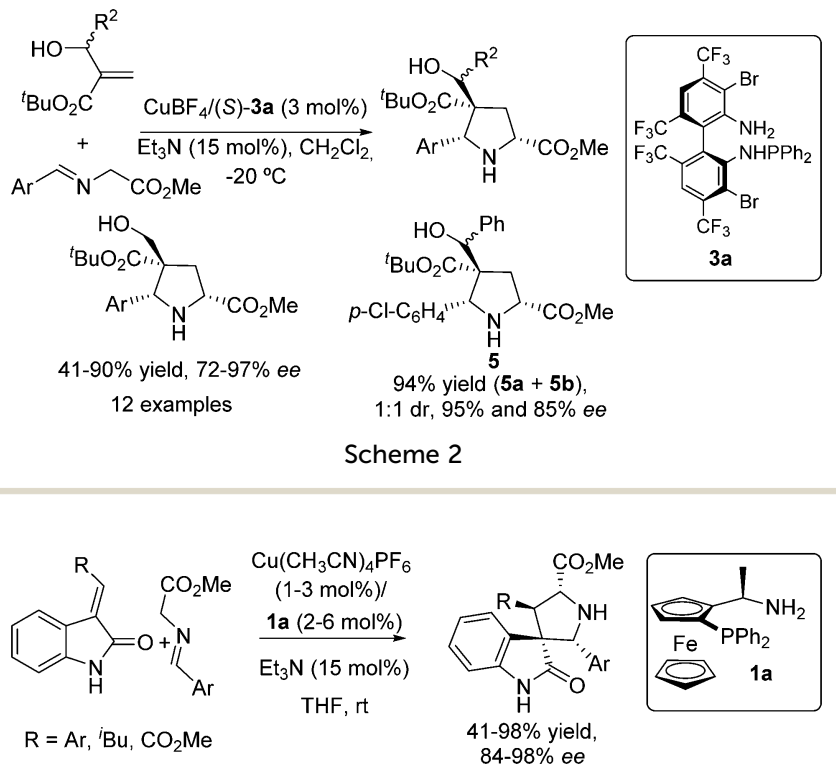

Scheme 3

Pyrrolidines bearing spiroquaternary stereogenic carbon centres have attracted great attention due to their interesting biological properties. Since the seminal work of Waldmann ${ }^{13}$ (Scheme 3), describing the preparation of 3,3-pyrrolidinyl spirooxoindole derivatives, the 1,3-dipolar cycloaddition of azomethine ylides has emerged as an essential tool for the preparation of spirocyclic pyrrolidines.

In the aforementioned Waldmann's work the exo cycloadducts were exclusively obtained catalyzed by the $\mathrm{CuPF}_{6}\left(\mathrm{CH}_{3} \mathrm{CN}\right)_{4} /$ $\mathrm{N}, \mathrm{P}$-ferrocenyl ligand $\mathbf{1}$ complex. In a later report a similar diastereoisomeric outcome was observed by Wang and co-workers using AgAcO/TF-Biphamphos 3a. ${ }^{14}$

In 2010 Arai and co-workers reported the first example of exo-selective cycloaddition of azomethine ylides (with nitrostyrenes as dipolarophiles) to provide 2,5-trans configured pyrrolidines. ${ }^{15}$ This atypical stereochemical behavior was observed using the $\mathrm{Ni}(\mathrm{OAc})_{2}$ /ligand 6 complex as the catalyst. The extension of this methodology to the [3+2] cycloaddition of methyleneindolinone allowed the preparation of exo spirooxoindoles with excellent selectivities ${ }^{16}$ (Scheme 4).

In 2011 Wang and co-workers ${ }^{17}$ described the preparation of spirocyclic pyrrolidines by 1,3-dipolar cycloaddition of azomethine ylides with sterically hindered $\alpha, \alpha, \beta$-trisubstituted alkylidene cycloketones catalysed by copper TF-Biphamphos complexes. Silver(I) salt

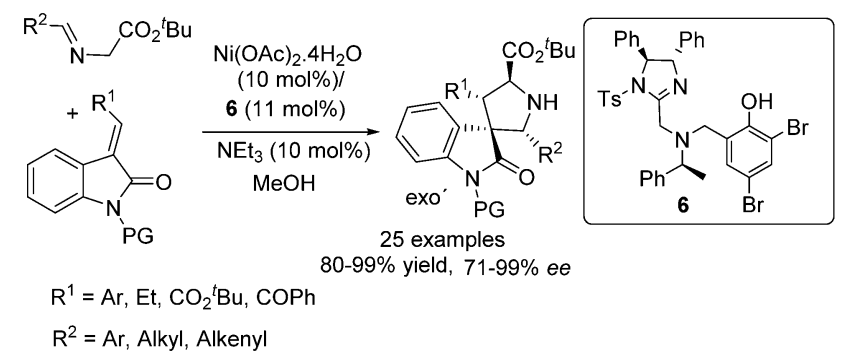

Scheme 4 

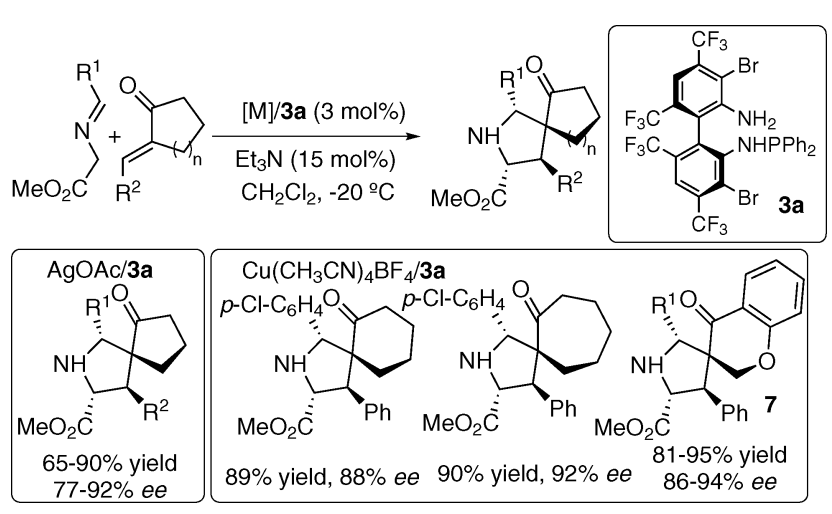

Scheme 5

gave better results that copper(I) when 2-benzylidenecyclopentenone was used as dipolarophile, furnishing the desired spirocyclic pyrrolidines with excellent diastereoselectivity and up to $92 \%$ ee (Scheme 5). In contrast, the $\mathrm{Cu}^{\mathrm{I}} / \mathrm{TF}-$ Biphamphos $\mathbf{3 a}$ complex afforded better enantioselectivities for cyclohexanone and cyclopentenone derivatives. The further regioselective Baeyer-Villiger oxidation of the adducts provided the enantioselective access to synthetically useful pyrrolidinyl spirolactones. An extension of this work, also described in $2011,{ }^{18}$ explored the reaction of 3-alkylidene-4-chromanones with iminoesters to give spiro-4chromanones-3,3'-pyrrolidine derivatives 7 (Scheme 5).

In 2013 Wang's group ${ }^{19}$ also studied the use of $\alpha$-methylene butyrolactones as dipolarophiles. A variety of spirocyclic butyrolactone pyrrolidines bearing a quaternary stereogenic center were prepared in high yield and excellent stereoselectivity using the $\mathrm{CuBF}_{4} / \mathrm{DTBM}$-Biphep 8 complex as the catalyst. Interestingly, cyclic iminoesters also gave satisfactory results providing a tricyclic skeleton bearing two spirolactone units (Scheme 6).

One of the main synthetic limitations of this kind of cycloaddition is that it is only applicable to low LUMO dipolarophiles, mainly electron-deficient conjugated alkenes, hampering the preparation of pyrrolidines with electron-donating substituents at C-3 or C-4 positions of the pyrrolidine ring. The first approaches to overcome this limitation, involving $\alpha$-heteroatom substituted activated alkenes, have been recently described. In 2013 our research group reported the utilization of alkylidene azlactones as alkene partners in catalytic asymmetric 1,3-dipolar cycloadditions of azomethine ylides. $^{20}$ The use of the Ag/DTBM-Segphos 4a complex as a catalyst led to the formation of the desired spirocyclic intermediate 9 that due to its instability was quantitatively transformed into the corresponding dimethyl-4benzamidopyrrolidine-2,4-carboxylates $\mathbf{1 0}$ by in situ treatment

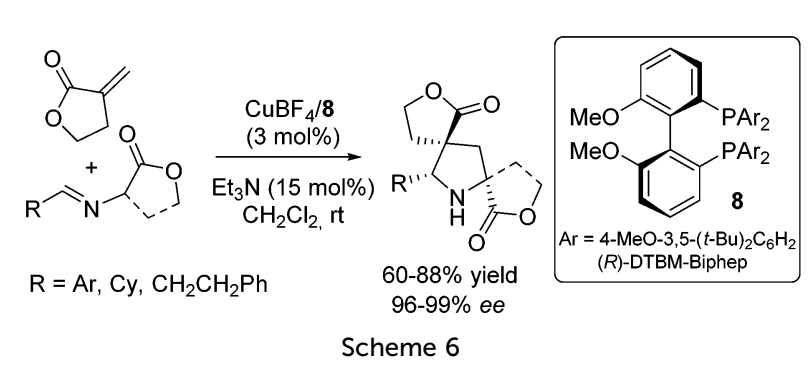

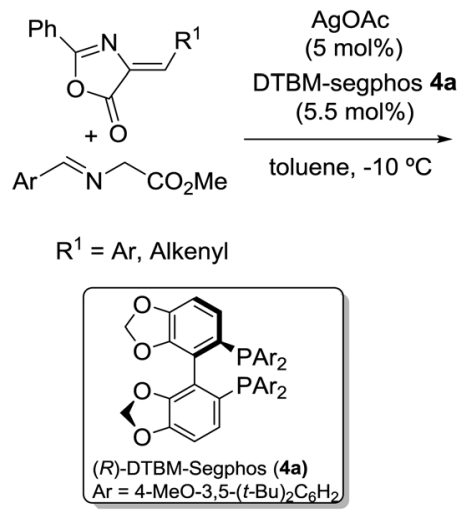

Scheme 7

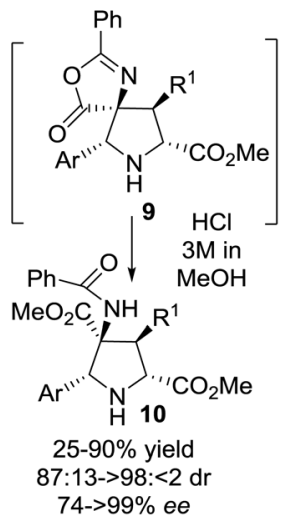

with $\mathrm{HCl}$ in methanol. Excellent levels of diastereoselectivity and enantiocontrol (up to $99 \%$ ee) were obtained for a variety of aromatic iminoesterers and aryl and alkenyl azlactones (Scheme 7).

Almost simultaneously with the publication of the aforementioned work, Deng and co-workers ${ }^{21}$ reported the synthesis of 4-aminopyrrolidine-2,4-dicarboxylic acid derivatives 11 via 1,3-dipolar cycloaddition of iminoesters with $\alpha$-phthalimidoaminoacrylates 12. After establishing the optimal conditions (AgOAc 5 mol\%, N,P-ligand 135.5 mol\%, $\mathrm{K}_{2} \mathrm{CO}_{3} 2.0$ equiv., CPME, $4 \AA$ A $\mathrm{MS}$ at $40{ }^{\circ} \mathrm{C}$ ) a survey of $\alpha$-iminoesters were tested in the reaction providing the corresponding pyrrolidines with high yield and selectivity (Scheme 8). Remarkably, $\beta$-substituted $\alpha$-phthalimidoaminoacrylates also proved to be suitable substrates in this transformation, allowing the preparation of tetrasubstituted pyrrolidines.

Very recently, Waldmann, Antonchick and co-workers ${ }^{22}$ have developed a catalytic enantioselective 1,3-dipolar cycloaddition between $\alpha$-iminoesters and $2 H$-pyran-3(6H)-ones. In the presence of $\mathrm{Cu}\left(\mathrm{CH}_{3} \mathrm{CN}\right)_{4} \mathrm{BF}_{4}(5 \mathrm{~mol} \%)$, Fesulphos 2 (6 mol\%), and DBU (20 mol\%) an efficient kinetic resolution of racemic pyranones occurred, providing the desired cycloadducts with high diastereo and enantioselectivity (Scheme 9). The method showed a wide substrate scope, highlighted with the preparation of more than one hundred pyrrolidine adducts. The biological evaluation of this compound, which encompasses structural similarity with the biologically active naturally occurring iridoids, led to the discovery of a new group of inhibitors of the $\mathrm{Wnt}^{23}$ and Hedgehog signalling. ${ }^{24}$

In spite of allenes being widely applied in Diels-Alder and 1,3-dipolar cycloaddition reactions, their use in catalytic asymmetric

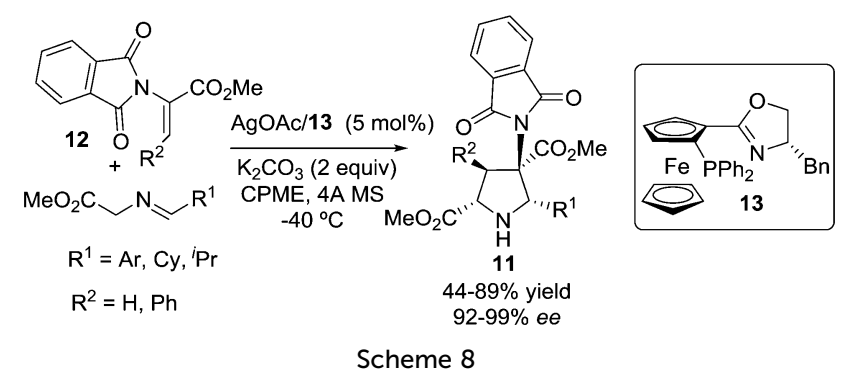




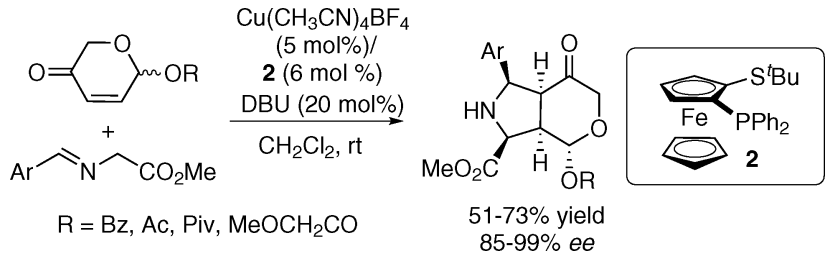

Scheme 9

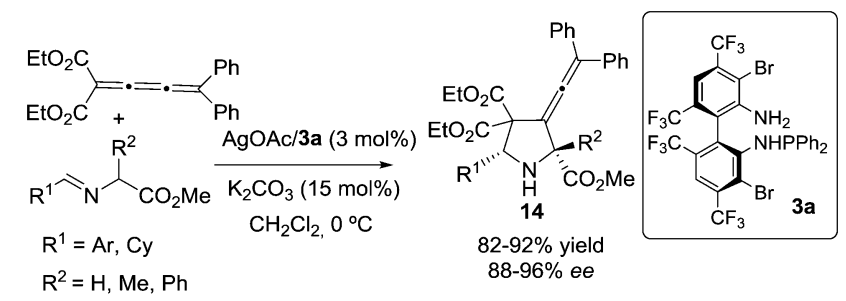

Scheme 10

1,3-dipolar cycloaddition of azomethine ylides was not reported until recently.

In 2011 Wang and co-workers reported that the AgOAc/TF Biphamphos 3a complex efficiently catalyzed the 1,3-dipolar cycloaddition of azomethine ylides with diethyl 2-(3,3-diphenylpropa-1,2-dienylidene)malonate. ${ }^{25} 3$-Vinylidene-pyrrolidines 14 were obtained with high enantioselectivity, including the reaction with alanine or phenylglycine derived iminoesters (Scheme 10).

\subsection{Fluorinated dipolarophiles}

The introduction of a fluorine atom into organic compounds usually has an important effect in their biological properties. Therefore, the widespread presence of the pyrrolidine ring in many biologically active compounds has stimulated the preparation of fluoropyrrolidine derivatives. However, most of the current synthetic methods are based on chiral starting materials rather than catalytic asymmetric approaches. In this context our research group described in 2007, while conducting studies on 1,3-dipolar cycloaddition of $\alpha, \beta$-unsaturated sulfones, the catalytic asymmetric cycloaddition of trifluoromethyl vinyl sulfone with glycine iminoesters. ${ }^{26}$ In 2011 Wang and co-workers ${ }^{27}$ developed a catalytic asymmetric procedure for the preparation of trifluoromethylated pyrrolidines using trans-trifluorocrotonates as dipolarophiles. The 1,3-dipolar cycloaddition with glycine iminoesters proceeded smoothly using the combination of $\mathrm{Cu}\left(\mathrm{CH}_{3} \mathrm{CN}\right)_{4} \mathrm{BF}_{4} / \mathrm{TF}$ Biphamphos $3 \mathrm{a}$ as a catalytic system to give the corresponding adducts endo-15 with excellent endo-diastereoselectivity and enantioselectivity (Scheme 11). $Z$-Isomers are also suitable substrates in this cycloaddition. Thus, the reaction of cis-4,4,4-ethyl trifluorocrotonate under the optimized conditions afforded the endo cycloadduct 16 in good yield and enantioselectivity. In a further study the exo adducts $\mathbf{1 5}$ were selectivity obtained in the presence of the bulky DTBM-Biphep ligand $\mathbf{8}^{28}$

In 2012 the same research group reported the preparation of pyrrolidines bearing a fluorinated quaternary stereocenter using methyl $\alpha$-fluoroacrylate $\mathbf{1 7}$ as a dipolarophile. ${ }^{29}$ Again the $\mathrm{Cu}\left(\mathrm{CH}_{3} \mathrm{CN}\right)_{4} \mathrm{BF}_{4} / \mathrm{TF}$ Biphamphos 3a system afforded the best
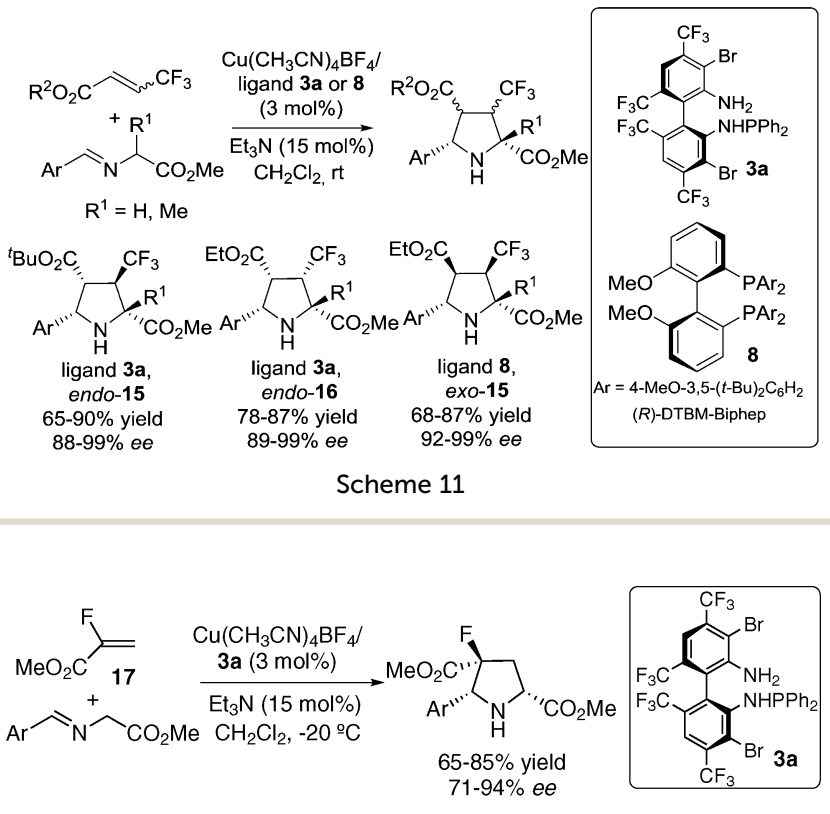

Scheme 12

performance. The reaction with a variety of glycinate iminoesters provided the fluorinated pyrrolidines with high stereoselectivities (Scheme 12).

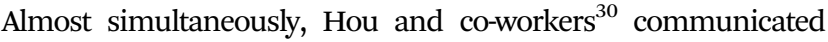
the preparation of 3-(fluoromethyl)-4-nitroproline derivatives by 1,3-dipolar cycloaddition with fluoromethyl substituted nitroalkenes. In this case, the combination of $\mathrm{CuClO}_{4}$ and Walphos ligand 18 afforded the best results, allowing the access to substituted exo adducts with high diastereoselectivity and enantioselectivity. The use of LiHMDS as base, instead of $\mathrm{Et}_{3} \mathrm{~N}$, led to a significant improvement of the reactivity (Scheme 13). The reduction of the nitro group afforded 3-trifluoromethyl-4-amino pyrrolidines with potential biological interest.

The 1,3-dipolar cycloaddition of azomethine ylides with imines is a very interesting approach to the direct synthesis of imidazolines. ${ }^{31}$ However, the lower reactivity of imines in comparison with typical electron deficient olefins has limited the scope of this transformation. Fluorinated imines have been employed to enhance the reactivity of this cycloaddition. Thus, in $2010 \mathrm{Wu}$ and co-workers ${ }^{32}$ described the silver catalyzed 1,3-dipolar cycloaddition of azomethine ylides with fluorinated imines. The corresponding tetrahydroimidazole derivatives were obtained with high yield and diastereoselectivity. The enantioselective version of this process was reported in 2013 by the group of Wang using the complex Cu-PPFOMe 19 as the catalyst. $^{33}$ The reaction showed a broad substrate scope and

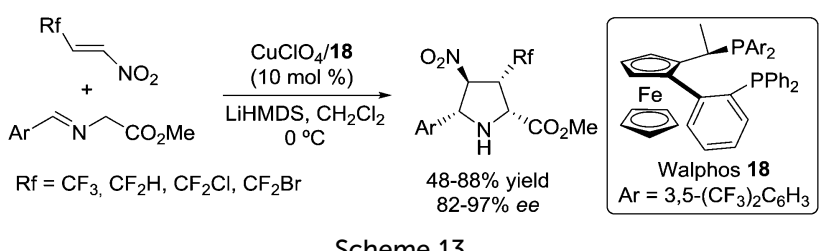

Scheme 13 


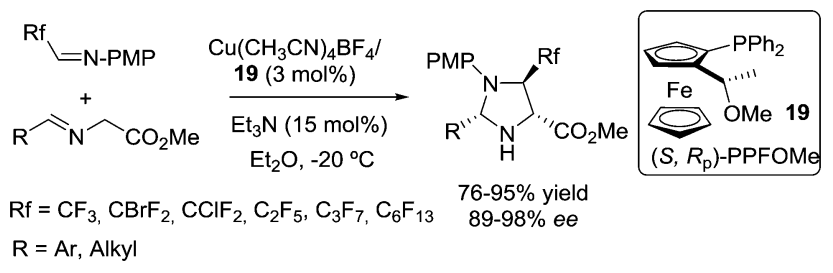

Scheme 14

provided the imidazoline adducts with high diastereoselectivity and enantioselectivity (Scheme 14).

\subsection{Quinones as dipolarophiles}

Quinones and their derivatives have found a wide range of applications in organic synthesis and have been extensively applied in Diels-Alder and 1,3-dipolar cycloadditions. ${ }^{34}$ However, their use as dipolarophiles in catalytic asymmetric [3+2] cycloaddition with azomethine ylides has been seldom explored.

In 2012 Antonchick, Waldmann and co-workers ${ }^{35}$ developed a catalytic asymmetric tandem reaction of azomethine ylides with $p$-benzoquinone. Two consecutive cycloadditions led to structurally complex molecular scaffolds with up to eight stereocenters. In the presence of catalytic amounts of $\mathrm{Cu}^{\mathrm{I}}$ salt and Fesulphos ligand the reaction proceeded with good yield and high regioselectivity, diastereoselectivity and enantioselectivity, allowing the formation of one stereoisomer from 512 possible isomers. The chirality of the ligand controls the enantioselectivity of the first cycloaddition (enantioselectivity-determining step) while the stereoselectivity of the second cycloaddition is directed by the stereogenic centres formed in the first step. Therefore, since the enantioselectivity is exclusively controlled in the first step of the sequence, both enantiomers can be selectively obtained using the same chiral ligand simply by changing the order of the iminoester ( 20 and 21) additions (Scheme 15).

Also in 2012 Wang and co-workers ${ }^{36}$ described the synthesis of highly enantioenriched isoindolines by copper catalyzed enantioselective 1,3-dipolar cycloaddition of azomethine ylides with naphthoquinone or benzoquinone and subsequent silica gel promoted aromatization. $\alpha$-Substituted iminoesters were used to avoid the undesired oxidation to pyrrole derivatives (Scheme 16).

Desymmetrization of the prochiral cyclohexadienone moiety is an efficient tool for the enantioselective preparation of structurally and stereochemically complex compounds. Wang and co-workers described the reaction of prochiral spirocyclohexadione 22 and iminoesters catalyzed by AgOAc/TF-Biphamphos $3 \mathbf{b}^{37}$ The corresponding spiro heterocyclic product $\mathbf{2 3}$ was obtained as the sole isomer in good yield and excellent enantioselectivity. The reaction showed a wide substrate scope regarding both the iminoester and the spirolactone partners (Scheme 17).

\subsection{Fullerenes as dipolarophiles}

Fullerenes are considered to be very useful organic scaffolds with potential applications in several scientific areas. ${ }^{38}$ Therefore, the preparation of chiral enantioenriched fullerenes is a major goal since the stereochemical configuration exerts a great
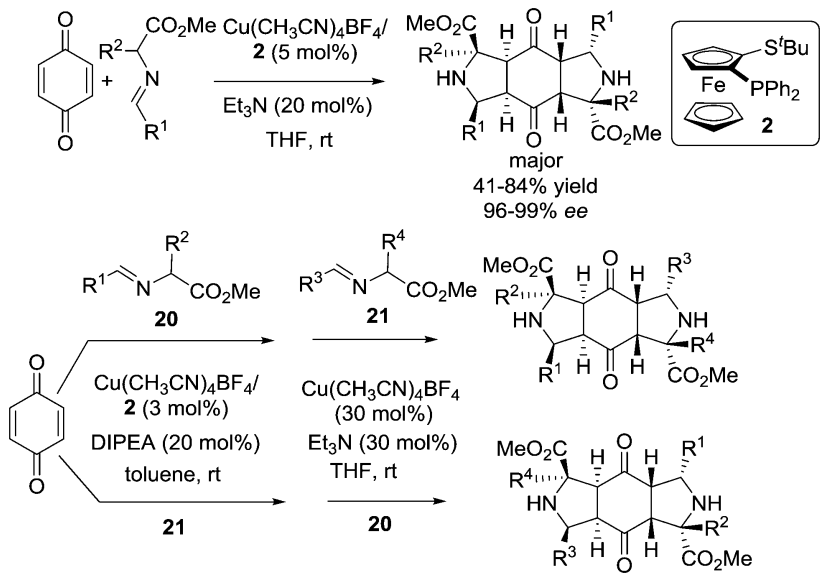

Scheme 15

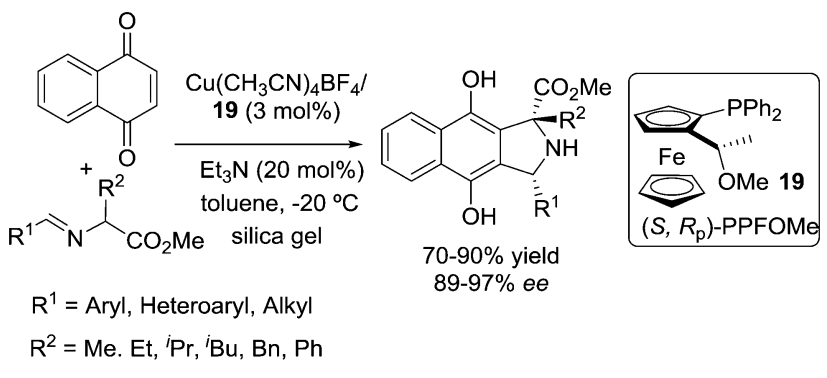

Scheme 16
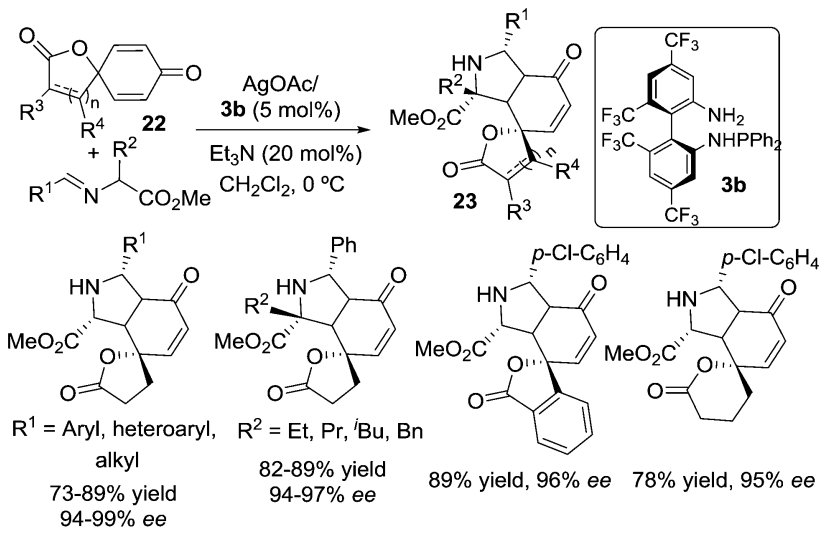

Scheme 17

influence on some fullerene physical properties. ${ }^{39}$ However, until recently, the lack of efficient synthetic methods for the enantioselective preparation of chiral fullerenes has hampered their applicability.

In this context, the 1,3-dipolar cycloaddition of azomethine ylides has emerged as one of the most straightforward procedures for the functionalization of fullerenes. ${ }^{40}$ In 2009 Martín and co-workers reported the first catalytic asymmetric example of this transformation. ${ }^{41}$ The combination of copper(II)/Fesulphos ligand or silver(I)/BPE ligand catalyzed the reaction of iminoesters with C60 leading to the formation of the corresponding cis-adducts with high yield and excellent enantioselectivity. Using $\mathrm{Cu}^{\mathrm{II}} / \mathrm{binap}$ 


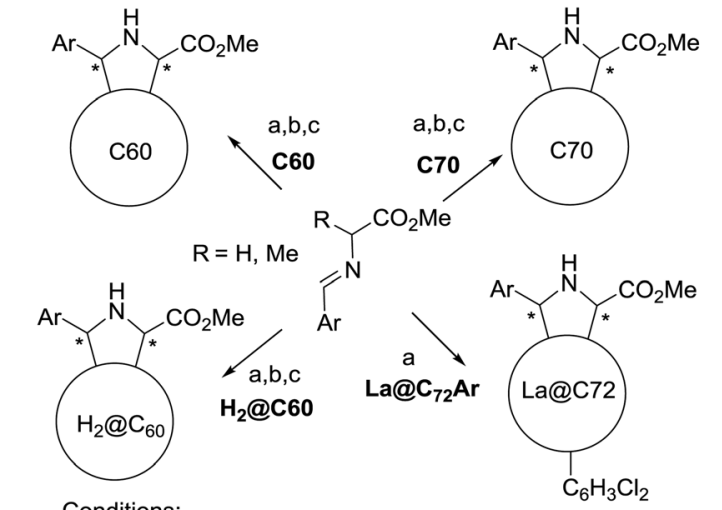

c60:

Conditions:

a: $\mathrm{Cu}(\mathrm{OAc})_{2} /$ Fesulphos, cis $95->99 \%$, 65-93\% ee; b: AgOAc/BPE, cis $80-$ $>99 \%, 70-90 \%$ ee; $\mathrm{c}: \mathrm{Cu}(\mathrm{OAc})_{2} / \mathrm{DTBM}-\mathrm{Segphos}$, trans $72-95 \%, 91-97 \%$ ee (opposite enantiomer)

C70:

a: regioselectivity : $82: 18-90: 10$, cis $95-99 \%, 92-99 \%$ ee; b: regioselectivity : 81:19-90:10, cis $94-98 \%, 80-89 \%$; c: trans $80-99 \%, 90-95 \%$ ee

regioselectivity : $65: 35-97: 3$.

$\mathrm{H}_{2} @ \mathbf{C 6 0}$

a: cis, $94 \%$ ee; b: cis, $90 \%$ ee (opposite enantiomer); c: trans $94 \%$ ee La@ $\mathrm{C}_{72} \mathrm{C}_{6} \mathrm{H}_{3} \mathrm{Cl}_{2}$ racemic mixture:

a: eight isomers are formed, separated by HPLC

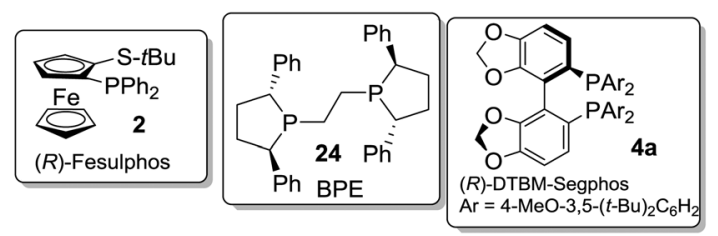

Scheme 18

as the catalyst, trans pyrrolidinofullerenes were mainly obtained albeit with modest enantioselectivity (20-40\% ee). In a later study trans pyrrolidines were also selectively obtained with DTBMSegphos $4 \mathbf{a}$ as the chiral ligand. ${ }^{42}$ This methodology was subsequently applied to the preparation of C70 derivatives which encompass four different types of double bonds, and therefore selectivity control is more challenging. ${ }^{43}$ In this case AgOAc/ diphenylphospholono $\mathbf{2 4}$ or $\mathrm{Cu}(\mathrm{OAc})_{2} /$ Fesulphos 2 complexes led to the corresponding adduct with high site-(only $\alpha$ isomer) regio-, diastereo-, and enantioselectivity (Scheme 18). This methodology has been subsequently applied to the preparation of chiral endohedral fullerenes. Excellent stereoselectivities were achieved in the cycloaddition of $\mathrm{N}$-metalated azomethine ylides with endohedral fullerene $\mathrm{H}_{2} @ \mathrm{C}_{60} \cdot{ }^{44}$ The reaction of a racemic mixture of a metallofullerene derivative, La@C $72\left(\mathrm{C}_{6} \mathrm{H}_{3} \mathrm{Cl}_{2}\right)^{45}$ with azomethine ylides catalyzed by the $\mathrm{Cu}^{\mathrm{II}} /$ Fesulphos 2 complex afforded a mixture of eight isomers (Scheme 18).

\subsection{Higher order cycloadditions}

As shown in all the previous examples, the 1,3-dipolar reaction of azometine ylides is a well established method for the preparation of five membered rings via [3+2] cycloaddition. In contrast, the preparation of larger rings by catalytic asymmetric higher order processes has been seldom explored. This approach constitutes a challenge which would open the door

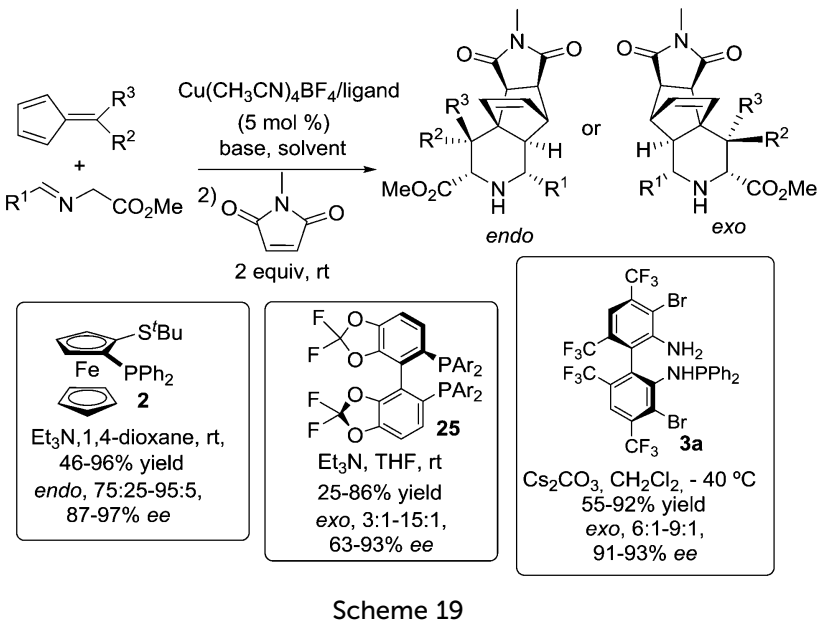

to the enantioselective preparation of medium sized heterocycles in a single synthetic operation.

[6+3] cycloadditions. In 2003 Hong and co-workers developed a stereoseolective silver catalyzed [6+3] cycloaddition of iminoesters to fulvenes to give piperidine derivatives with high yield and diastereoselectivity. ${ }^{46}$ In 2012 Antonchick and Waldmann reported the enantioselective version of this process. ${ }^{47}$ The best results were obtained using the combination of $\mathrm{Cu}\left(\mathrm{CH}_{3} \mathrm{CN}\right)_{4} \mathrm{BF}_{4}$ and Fesulphos ligand 2 as a catalyst system. Due to the low stability of the adducts a subsequent Diels-Alder reaction between the cyclopentadiene moiety and $N$-methylmaleimide was performed. The [4+2] cycloaddition afforded only one diastereomer without any detectable epimerization. Interestingly, the scope of this $[6+3] /[4+2]$ one-pot sequence is very general with regard to the iminoester and fulvene substitution, and several dienophiles are compatible with the process (Scheme 19).

Almost simultaneously, Wang and co-workers ${ }^{48}$ reported a similar cycloaddition catalyzed by $\mathrm{Cu}^{\mathrm{I}} / \mathrm{TF}-\mathrm{Biphamphos}$ 3a. In contrast with Waldmann's procedure, the reaction is exo-selective giving rise to 3,4-trans piperidine as a sole isomer. The stereodivergent synthesis of exo-adducts was also completed by Waldmann's group using commercially available difluorophos ligand $25 .{ }^{49}$

Tropone derivatives have been widely studied as reaction partners in higher order cycloadditions. ${ }^{50}$ However, only a few examples regarding 1,3-dipolar cycloadditions of troponoids with dipoles have been documented.

Very recently, the first metal catalyzed [6+3] cycloaddition of tropone with azomethine ylides has been developed. The reaction proceeded smoothly in the presence of $\mathrm{Cu}\left(\mathrm{CH}_{3} \mathrm{CN}\right)_{4} \mathrm{ClO}_{4}$ and ligand 26, providing piperidine fused bicyclic heterocycles with high diastereoselectivity and enantioselectivity. ${ }^{51}$ Concurrently, the same type of cycloaddition was reported by Wang and co-workers. In this case the $N$-monosubstituted ligand 27 afforded better results than the disubstituted chiral ligand 26. Interestingly, less reactive aliphatic iminoesters provided excellent results in the presence of $\mathrm{Cs}_{2} \mathrm{CO}_{3}$ as base (Scheme 20). ${ }^{52}$

[3+3] cycloadditions. The synthetic potential of cycloaddition reactions between two different types of 1,3-dipoles, including some catalytic asymmetric examples, has been recently demonstrated. ${ }^{53}$ 

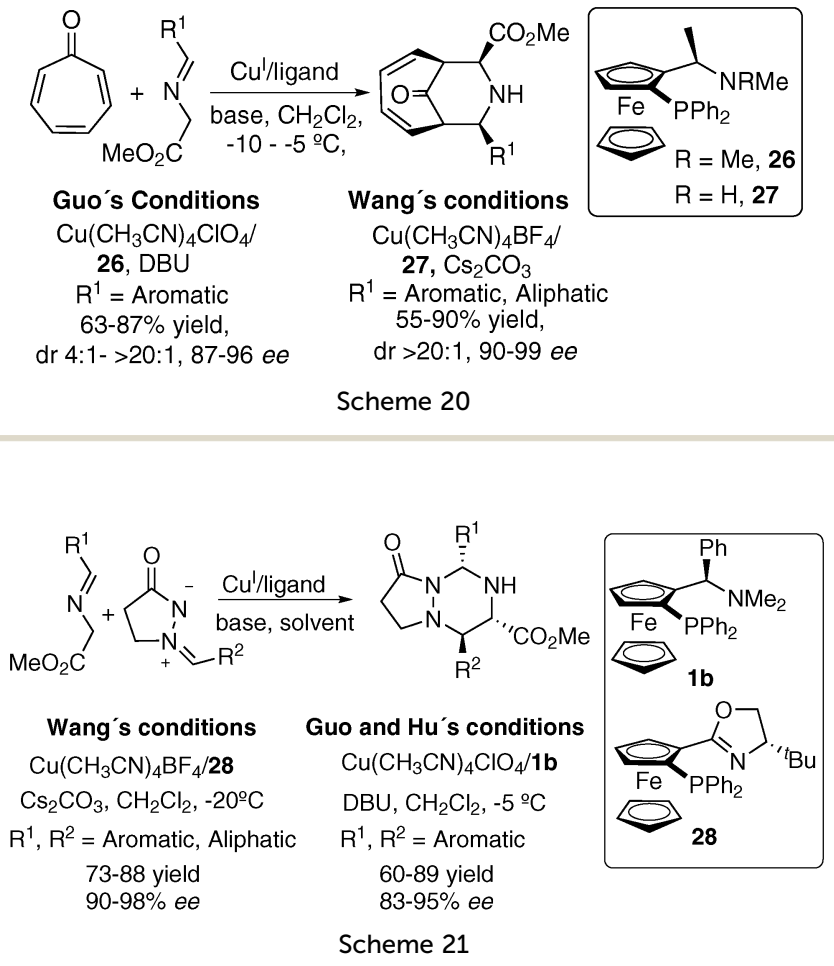

In the case of azomethine ylides the first asymmetric precedent was described in 2013 by Wang and co-workers, who described the catalytic asymmetric [3+3] cycloaddition of azomethine ylides with azomethine imines catalyzed by $\mathrm{Cu} /$ ferrocenyl ligand $28 .{ }^{54}$ A variety of azomethine ylides and azomethine imines, including those derived from aliphatic aldehydes, underwent the reaction to furnish the corresponding heterocyclic products in high yield and enantioselectivity. This process constitutes a very practical method for the enantioselective preparation of 8-oxohexahydro$6 H$-pyrazolo[1,2-a][1,2,4]triazine-3-carboxylate derivative skeletons, which are found in several biologically active molecules. Almost simultaneously Guo, $\mathrm{Hu}$ and co-workers described a similar procedure with Ugi amine derived ligand $\mathbf{1 b}$ and DBU as base $\mathrm{s}^{55}$ (Scheme 21).

\section{New azomethine ylide precursors in metal-catalyzed 1,3-dipolar cycloadditions}

As mentioned previously, $\alpha$-iminoesters derived from $\alpha$-amino acids (mainly glycine) are, by far, the most common dipole precursors in catalytic asymmetric 1,3-dipolar cycloadditions, providing pyrrolidines with 2-carboxylate substitution.

From 2007 to 2011 the first examples of other types of azomethine ylide precursors were reported. Thus, azlactones, ${ }^{56}$ $\alpha$-iminophosphonates, ${ }^{57} \alpha$-iminonitriles, ${ }^{58}$ and $N$-(2-pyridylmethyl)imines $^{59}$ were added to the arsenal of suitable partners for this reaction. Since 2011 the scope of the reaction with regard to the dipole has been significantly extended, as summarized next.

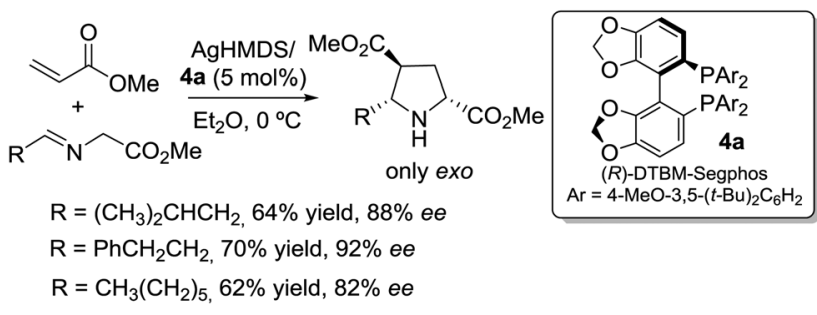

Scheme 22

\subsection{Iminoesters derived from aliphatic aldehydes}

Despite the impressive development of the 1,3-dipolar cycloaddition using $\alpha$-iminoesters, there are still some limitations. In particular, the reaction is usually restricted to the more stable iminoesters derived from non-enolizable aldehydes (mainly aromatic and $\alpha, \beta$-unsaturated aldehydes). Consequently, examples using Schiff bases derived from non-branched aliphatic aldehydes are rare. $^{60}$

In 2011 Kobayashi and co-workers ${ }^{61}$ reported that the combination of silver bis(trimethylsilyl)amide (AgHMDS) and DTBM-Segphos quiral ligand $\mathbf{4 a}$ efficiently catalyzed the asymmetric 1,3-dipolar cycloaddition of several primary alkyl aldehyde Schiff bases with methyl acrylate (Scheme 22).

Very recently, Garner and co-workers have developed a procedure based on glycyl sultams as azomethine ylide precursors. ${ }^{62}$ The sultam group activates the $\alpha$-position of the Schiff base making possible the in situ generation of azomethine ylides derived from enolizable aldehydes. Using $\mathrm{Cu}^{\mathrm{I}} / \mathrm{DTBM}$-Segphos $\mathbf{4 a}$ as the catalyst, in the absence of any external base, a variety of 5-alkyl-pyrrolidines were efficiently prepared (Scheme 23). ${ }^{63}$

\subsection{Cyclic azomethine ylides}

In 2006, Grigg and co-workers ${ }^{64}$ described the synthesis of spirocyclic pyrrolidines via 1,3-dipolar cycloaddition of homoserine lactone derived aldimino esters with electron-deficient olefins. In 2012 Wang and co-workers ${ }^{65}$ reported the highly efficient catalytic enantioselective version of this process, using $\mathrm{Cu}^{\mathrm{I}} / \mathrm{TF}$-Biphamphos $3 \mathbf{a}$ as the catalyst, in the reaction with maleates (Scheme 24).

In 2013 Reisman and co-workers reported a very efficient procedure for the synthesis of pyrrolizidines via double 1,3-dipolar cycloaddition. ${ }^{66}$ The reaction of an iminoester and tert-butyl acrylate under Schreiber's conditions ${ }^{67}$ provided the pyrrolidine 29 with high yield and selectivity. The pyrrolizidine formation was achieved by subsequent in situ addition of cinnamaldehyde (1 equiv.), an additional dipolarophile (5 equiv.) and warming the mixture to $23{ }^{\circ} \mathrm{C}$. A variety of dipolarophiles can be used in the second

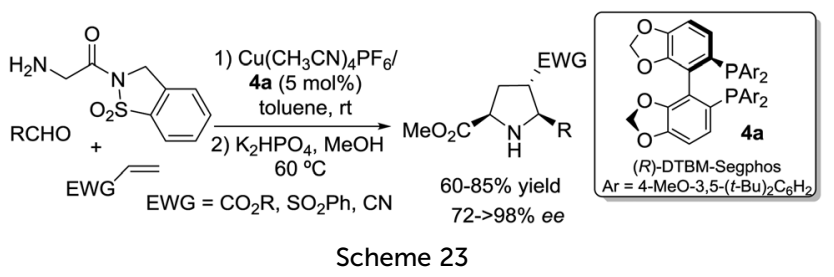

Scheme 23 


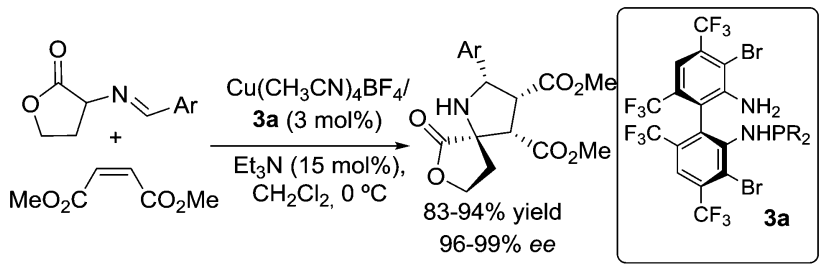

Scheme 24

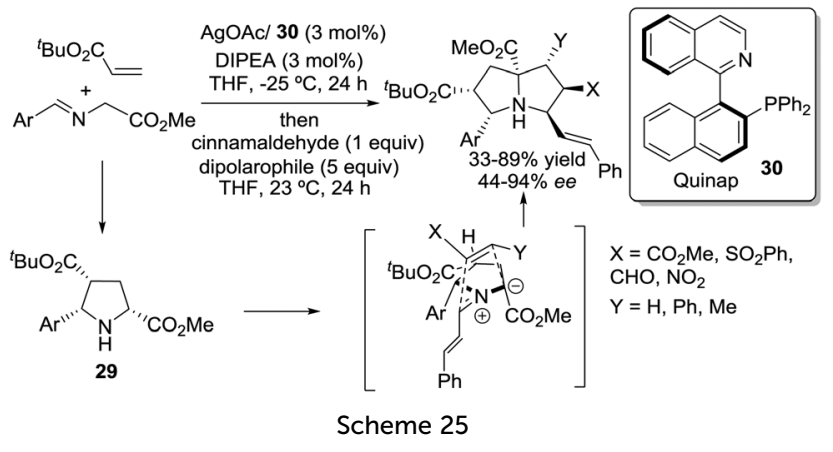

1,3-dipolar cycloaddition, affording the corresponding pyrrolizidines with high yields and enantioselectivities. However, only $\alpha, \beta$-unsaturated aldehydes are capable of forming the azomethine ylide needed for the second cycloaddition. The selectivity of the second cycloaddition is controlled by the stereogenic centres generated in the first cyclization (substrate control, Scheme 25).

Another general limitation of the catalytic asymmetric 1,3-dipolar cycloaddition is the low reactivity shown by the azomethine ylides derived from ketones. Very recently, Waldmann and co-workers ${ }^{68}$ have reported the first example of catalytic asymmetric cycloaddition of 1,3-fused cyclic azomethine ylides. In the reaction with nitrostyrenes the $\mathrm{Cu}^{\mathrm{I}}$-Fesulphos catalyst system showed the best performance affording the exo adducts with high diastereo and enantioselectivity. Remarkably, less reactive nitro derivatives such as $\beta, \beta$-disubstituted nitroalkanes also reacted with high enantioselectivity (Scheme 26). Interestingly, some of the resulting adducts showed activity as inhibitors of the Hedgehog-signaling cascade.

\subsection{Iminoamides as dipole precursors}

The expected strong metal coordination capacity of the carbonyl amide group, together with its chemical versatility and the biological interest of 2-amidopyrrolidines, ${ }^{69}$ makes $\alpha$-imidoamides excellent

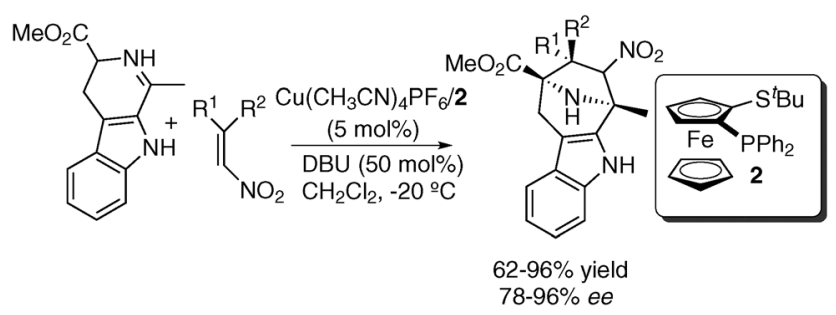

Scheme 26

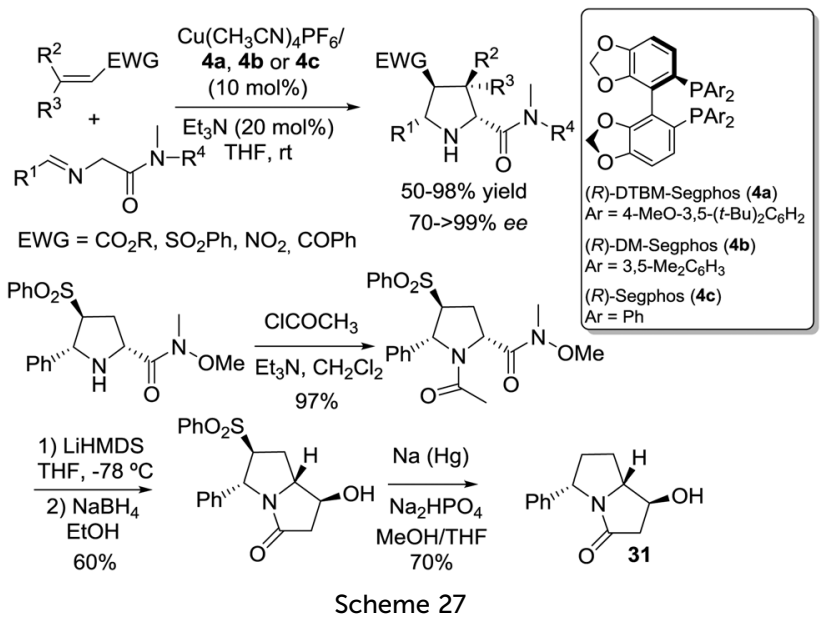

candidates as azomethine ylide precursors in 1,3-dipolar cycloadditions. Our research group has recently demonstrated the viability of this approach: ${ }^{70}$ Among the tested ligand the Segphos family afforded the best results, leading to the corresponding 2-amidopyrrolidines in excellent yield, exo-selectivity, and enantioselectivity. The reaction showed a wide structural scope with regard to both the $\alpha$-iminoamide and the dipolarophile, being compatible with several glycine-derived amides (including Weinreb amides) and activated alkenes. The synthetic usefulness of this methodology was also extended to the enantioselective synthesis of substituted pyrrolizidines 31 (Scheme 27).

\section{4. $\alpha$-Silylimines as dipole precursors}

$\alpha$-Silylimines were known to be suitable azomethine ylide precursors in non-asymmetric 1,3-dipolar cycloadditions, leading to racemic 5-unsubstituted pyrrolidines, a type of pattern not accessible via the classical process using $\alpha$-iminoesters. ${ }^{71}$ Their use in catalytic asymmetric processes has been documented very recently: in 2013 our research group demonstrated that the presence of a coordinating group in the $\alpha$-silylimine moiety enhances the reactivity and stereoselectivity of the process, likely by formation of a five membered metalated intermediate. Such a metallodipole would facilitate the asymmetric induction in the reaction with the dipolarophile.

Thus, the cycloaddition between $\alpha$-silylimines derived from $\alpha$-ketoesters and $N$-phenylmaleimide catalyzed by DTBMSegphos $/ \mathrm{Cu}^{\mathrm{I}}$ complexes provided the corresponding C-5 unsubstituted, C-2 quaternary substituted pyrrolidines in high yield and excellent enantiomeric excess. ${ }^{72}$ Excellent results were also obtained using $\alpha, \beta$-unsaturated sulfones as olefin partners. In this case quaternary proline derivatives were obtained after straightforward desulfonylation of the adducts (Scheme 28).

In a further work, other coordinating silylimines such as 2-pyridylsubstituted $\alpha$-silylimines were also explored. The reaction catalyzed by the $\mathrm{Cu}^{\mathrm{I}}$-Walphos $\mathbf{1 8}$ system led to the formation of $\alpha$-heteroarylpyrrolidines with high stereoselectivity. Interestingly, the use of water as an additive allowed to improve the reactivity of the reaction, the use of excess of the labile starting imine not being required (Scheme 29). ${ }^{73}$ 


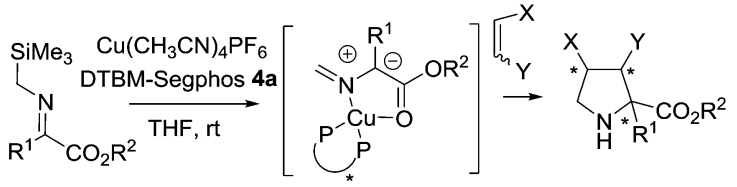<smiles>[R1][C@]1(C(=O)CC)NC[C@H]2C(=O)N(c3ccccc3)C(=O)[C@H]21</smiles>

$50-79 \%$ yield $81-98 \%$ ee

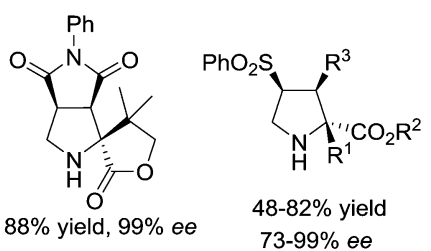

Scheme 28

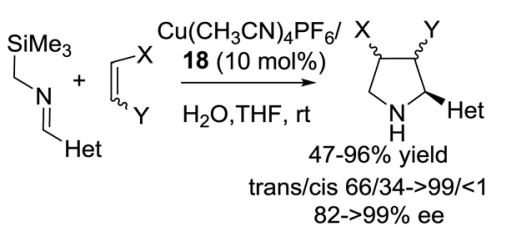

Het = 2-Py, 2-quinolyl, 2-thiazolyl

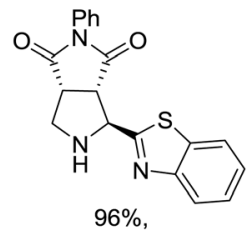

only $2,3-$ trans, $>99 \%$ ee

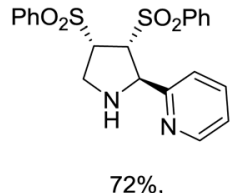

only 2,3-trans, $>99 \%$ ee

Scheme 29

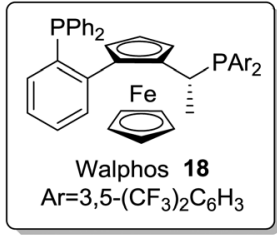

$\mathrm{O}_{2} \mathrm{~N}$

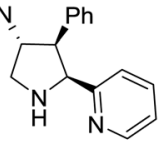

$71 \%$,

cis/trans $=90 / 10$

$94 \%$ ee (cis)

\section{Organocatalytic procedures}

The 1,3-dipolar cycloaddition also occupies a preeminent situation among the reactions amenable to asymmetric organocatalysis. Since the first report by MacMillan using nitrones, ${ }^{74}$ the range of suitable dipoles for organocatalytic processes has been significantly broadened.

The first organocatalytic asymmetric [3+2] cycloaddition of azomethine ylides, via activation of the dipolarophile by iminium ion formation, was reported by Vicario and co-workers in $2007 .^{75}$ Imines derived from diethyl aminomalonate smoothly reacted with $\alpha, \beta$ unsaturated aldehydes in the presence of $\alpha, \alpha$-diphenyl prolinol to afford the corresponding pyrrolidines with excellent enantiocontrol (Scheme 30, eqn (A)).

Afterward, Gong's ${ }^{76}$ and Chen's ${ }^{77}$ research groups independently described the cycloaddition between azomethine ylides and nitroalkenes catalyzed by a bifunctional thiourea scaffold (Scheme 30, eqn (B)).

In 2008 Gong and co-workers reported the first phosphoric acid catalyzed three component highly enantioselective dipolar cycloaddition between aldehydes, amino esters and activated olefins $^{78}$ (Scheme 30, eqn (C)). In recent years a great effort has been focused on developing novel asymmetric organocatalytic protocols.
Iminium activation (eq $A$ )

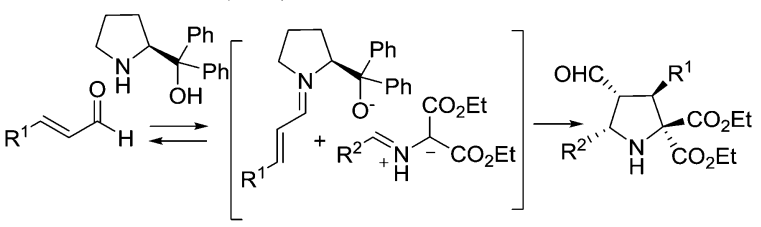

Thiourea activation (eq B)

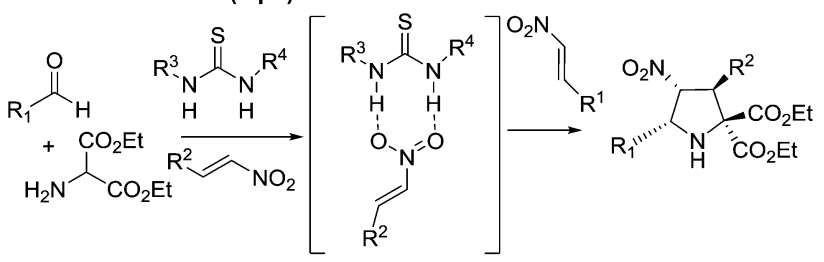

Brønsted acid activation (eq C)

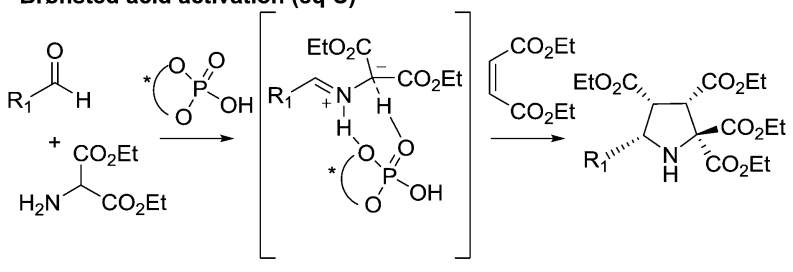

Scheme 30

\subsection{Iminium activation approach}

One of the usual structural requirements of the iminium activation protocol is the necessity of two electron-withdrawing groups at the azomethine ylide precursor in order to increase the acidity of the $\alpha$-hydrogen. The first examples of this approach were limited to the use of $\alpha$-iminomalonates.

In 2011, the groups of Vicario ${ }^{79}$ and Córdova ${ }^{80}$ independently reported the viability of using two different activating groups at the azomethine ylide precursor. Thus, the reaction of azomethine ylides derived from $\alpha$-iminoacetates with $\alpha, \beta$-unsaturated aldehydes led to the highly enantioselective synthesis of pyrrolidines owing a quaternary stereocenter at C-2 (Scheme 31).

Later Vicario and co-workers expanded this methodology to isoquinolinium and phthalizinium methylides. The cycloaddition catalysed by imidazoline $\mathbf{3 4}$ afforded the corresponding fused pyrrolidines with high diastereoselectivity and enantioselectivity ${ }^{81}$ (Scheme 32).

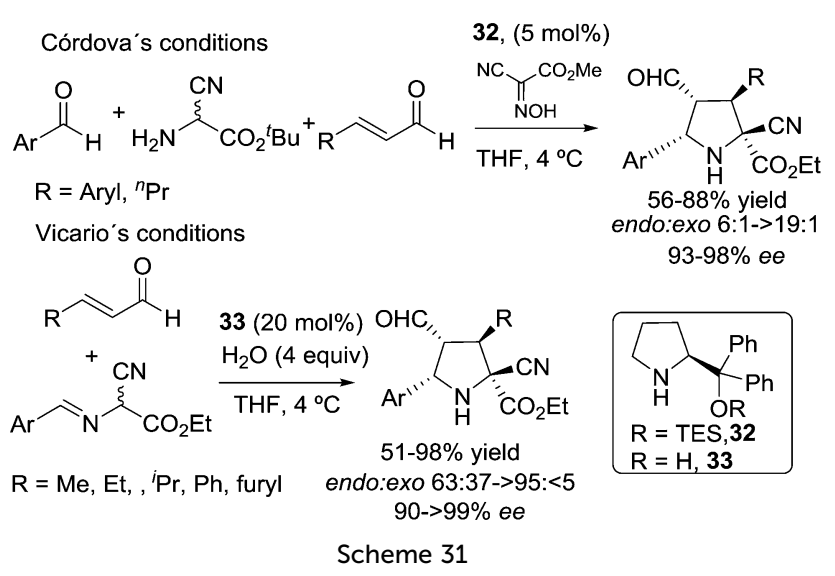




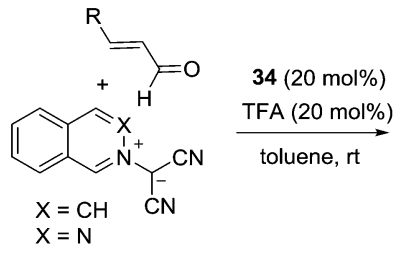

Scheme 32

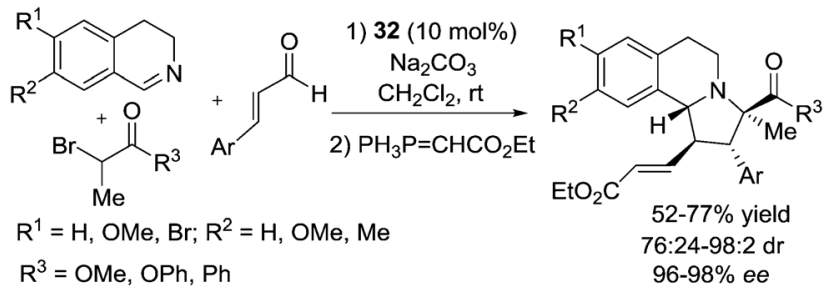

Scheme 33

The reaction of ylides generated in situ from 3,4-dihydroisoquinoline and $\alpha$-bromoesters (or $\alpha$-bromoketones) with $\alpha, \beta$-unsaturated aldehydes was reported by Jørgensen and co-workers. ${ }^{82}$ The reaction catalyzed by diphenyl-prolinol 32 proceeded with high enantioselectivity for a variety of aldehydes, imines and $\alpha$-bromo derivatives. The resulting adducts were directly transformed into $\alpha, \beta$-unsaturated esters by standard Wittig olefination (Scheme 33).

\subsection{Chiral Brønsted acid catalyzed processes}

The development of Brønsted acid catalyzed 1,3-dipolar cycloadditions has allowed us to significantly improve the scope of the reaction with regard to the dipolarophile.

Gong and co-workers have successfully applied the reaction to a series of electron-deficient alkenes. The reaction with 2,3-allenoates using phosphoric acid 35 as the catalyst proceeded smoothly to afford methylenpyrrolidines as single diastereomers with high enantioselectivity. ${ }^{83}$ This protocol has also been applied to the kinetic resolution of racemic 2,3-allenoates ${ }^{84}$ (Scheme 34).

Similarly, isoindoline derivatives were enantioselectively prepared by 1,3-dipolar cycloaddition using quinones as dipolarophiles and subsequent base mediated isomerisation ${ }^{85}$ (Scheme 35).

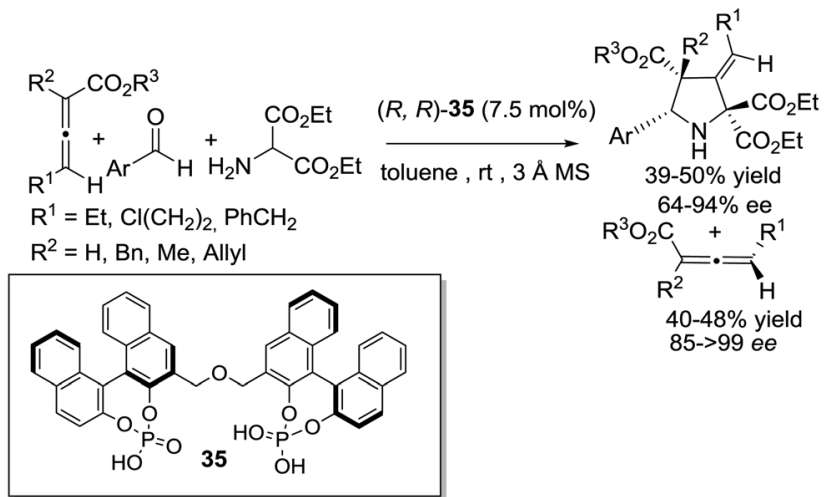

Scheme 34

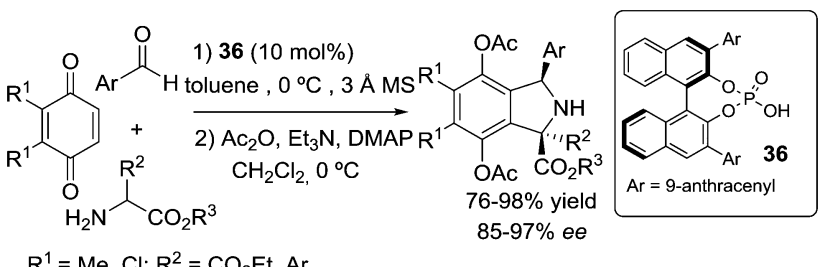

$\mathrm{R}^{1}=\mathrm{Me}, \mathrm{Cl} ; \mathrm{R}^{2}=\mathrm{CO}_{2} \mathrm{Et}, \mathrm{Ar}$

$\mathrm{R}^{3}=\mathrm{Me}, \mathrm{Et}$

Scheme 35

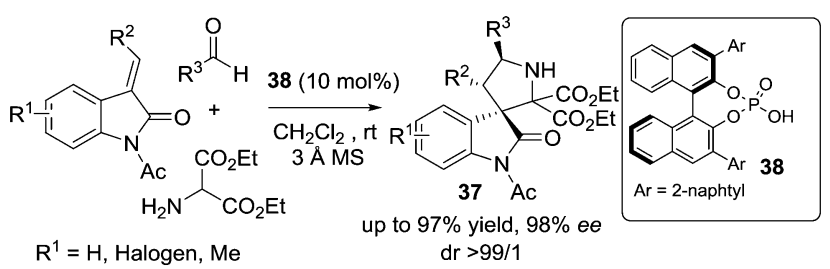

$R^{2}, R^{3}=$ Aryl, Alkyl

Scheme 36

Spiro[pyrrolidin-3,3,0-oxindole] derivatives were also prepared with high enantioselectivity and structural diversity using this methodology. ${ }^{86}$ Interestingly, the unusual regioisomer 37 was formed predominantly (Scheme 36).

The metal catalyzed asymmetric 1,3-dipolar cycloaddition of azomethine ylides and activated alkynes has remained elusive. This limitation has been recently overcome using Brønsted acid catalysis. The reaction catalyzed by phosphoric acid 36 furnished the corresponding 2,5-dihydropyrroles with excellent enantioselectivity $^{87}$ (Scheme 37).

$N$-Aryl imines are also suitable dipolarophiles for this transformation. The reaction with azomethine ylides catalyzed by phosphoric acid 39 provided the chiral imidazolidines with high stereoselectivity ${ }^{88}$ (Scheme 38 ).

Very recently the first example of organocatalyzed [3+3] cycloaddition has been reported by Shi and co-workers. ${ }^{89}$ The reaction of 3-indolylmethanol $\mathbf{4 0}$ with an in situ generated azomethine ylide catalyzed by the chiral phosphoric acid $\mathbf{4 1}$ afforded a variety of spiro-pyridoindoles with moderate diastereoselectivity and high enantiocontrol (Scheme 39).

As shown in all the previous examples, these protocols rely on imines derived from aminomalonates and aldehydes. The participation of other types of azomethine ylide precursors have been reported very recently.

The use of istatin based azomethine ylides was reported by Gong in 2012. ${ }^{90}$ This phosphoric acid catalyzed protocol

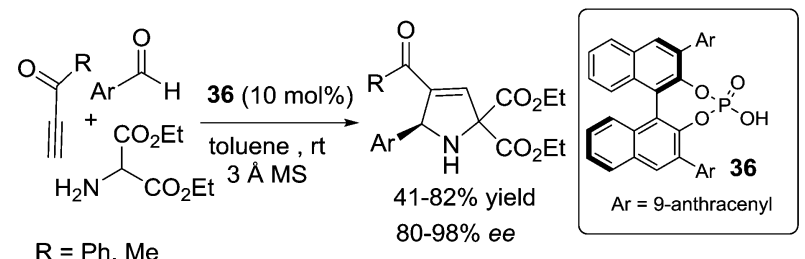

Scheme 37 


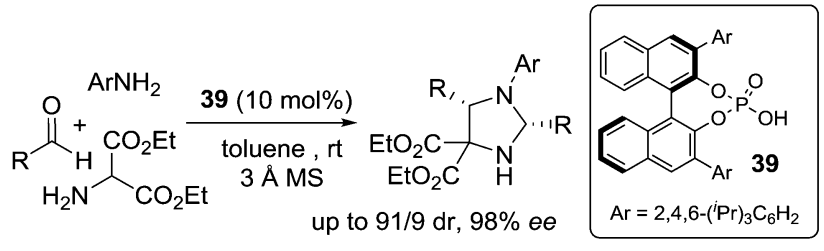

$\mathrm{R}=\mathrm{Ar}, \mathrm{c}-\mathrm{C}_{3} \mathrm{H}_{5}, \mathrm{Ph}=$

Scheme 38

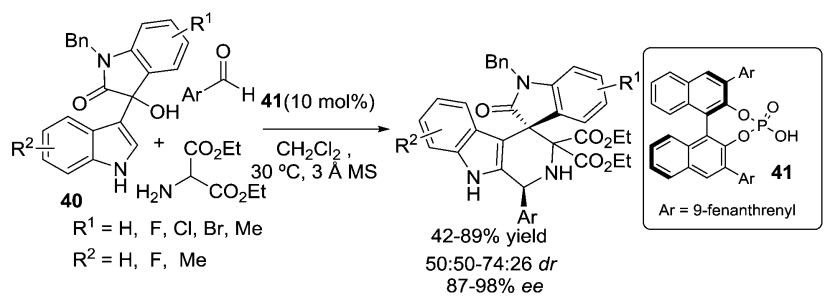

Scheme 39

allowed the preparation of highly functionalized spiropyrrolidin$3,2^{\prime}$-oxindoles 42 with excellent stereoselectivities and enantioselectivities with a variety of dipolarophiles (maleates, fumarates or phenylacrylate, Scheme 40). This methodology was later extended to activated alkynes as dipolarophiles. ${ }^{91}$

Gong and co-workers have also demonstrated that azomethine ylides can be formed from $\alpha$-keto esters and benzylamine by means of a biomimetic type transamination process. The cycloaddition with electron-deficient olefins afforded the pyrrolidine derivatives in high yields and excellent enantioselectivities (Scheme 41). ${ }^{92}$

\subsection{Thiourea activation strategies}

In $2011 \mathrm{Xu}$, Wang and co-workers described the cycloaddition between azomethine ylides and maleimides catalyzed by a tertiary bifunctional thiourea catalyst. ${ }^{93}$ Interestingly, the presence of two withdrawing groups is not required in this transformation. Thus, glycine derived iminoesters reacted smoothly with maleimides to afford the corresponding pyrrolidines with high stereocontrol (Scheme 42).

The first organocatalytic examples of 1,3-dipolar cycloaddition using azlactones (münchones) as azomethine ylide precursors have been recently reported. ${ }^{94}$ The reaction between $o$-hydroxy aromatic aldimines and alkylidene azlactones catalyzed by the bifunctional

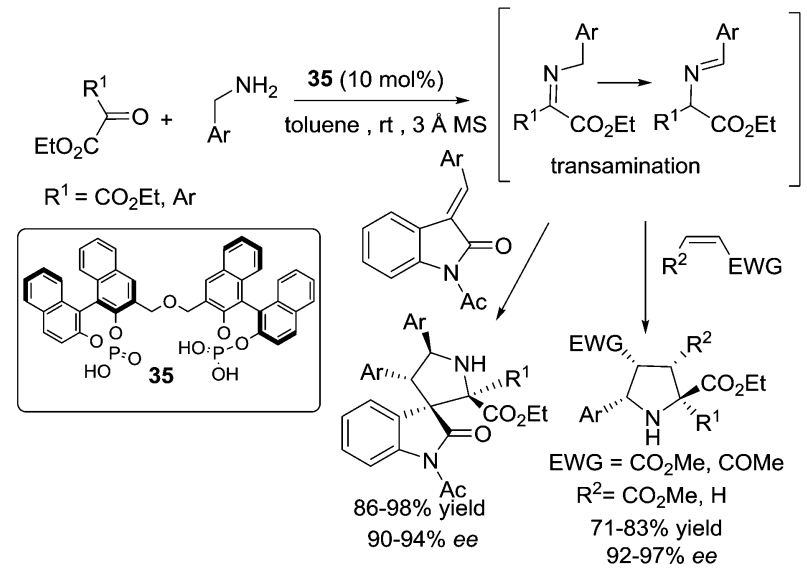

Scheme 41

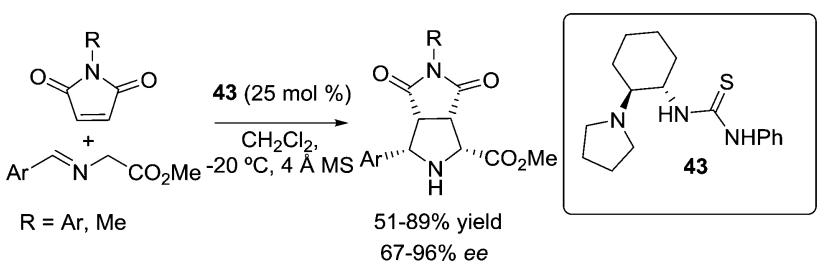

Scheme 42
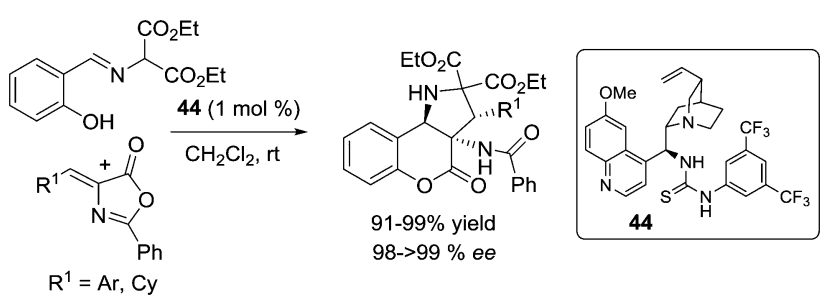

Scheme 43

thiourea 44 led to chromeno[4,3-b]pyrrolidine derivatives with excellent stereoselectivity under mild reaction conditions (Scheme 43).

Wang and co-workers ${ }^{95}$ reported the reaction of homoserine lactone derived aldimino esters with methyleneindolinone to afford highly functionalized tricyclic skeletons 45. Excellent levels of stereocontrol over the four contiguous stereocenters were achieved using a thiourea bifunctional organocatalyst 46 (Scheme 44).

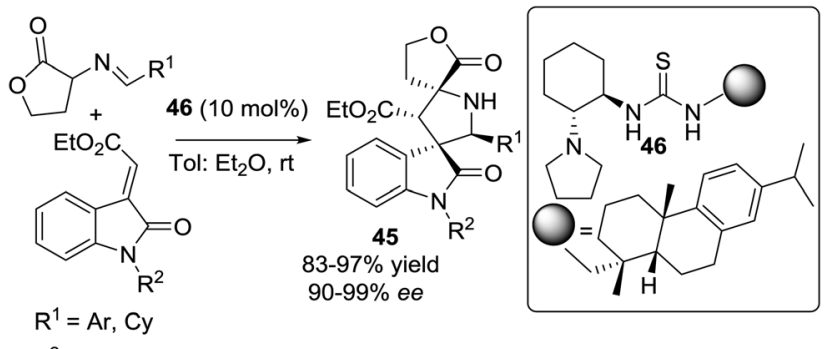

Scheme 44 


\section{Conclusions}

The development of a very efficient chiral catalyst for the enantioselective 1,3-dipolar cycloaddition of azomethine ylides has established this transformation as one of the most useful methodologies for the preparation of enantioenriched pyrrolidines. In recent years, a great deal of effort has focused on expanding the structural scope of this reaction with regard to both the azomethine ylide precursor and the dipolarophile. Thus, substituted and less reactive alkenes have been incorporated to the arsenal of suitable dipolarophiles. Similarly, the range of available dipole precursors has been widened from classical $\alpha$-iminoesters to a variety of different substituted imines. Interestingly, the first examples of higher order cycloaddition, allowing the preparation of six membered heterocycles have been developed. From a synthetic point of view, these achievements have greatly improved the synthetic applicability of this strategy opening the access to a wide variety of substituted pyrrolidine patterns and other types of substituted heterocycles.

\section{Acknowledgements}

The Ministerio de Economía y Competitividad (MINECO, project CTQ2012-3579) and Consejería de Educación de la Comunidad de Madrid (programme AVANCAT; S2009/PPQ-1634) are gratefully acknowledged for financial support. We also sincerely thank all the co-workers of our group involved in 1,3-dipolar cycloadditions.

\section{Notes and references}

1 (a) Cycloaddition Reactions in Organic Synthesis, ed. S. Kobayashi and K. A. Jørgensen, Wiley-VCH Verlag GmbH, Weinheim, Germany, 2002, pp. 5-326; (b) Pericyclic Reactions A Textbook: Reactions, Applications and Theory, ed. S. Sankararaman, Wiley-VCH Verlag GmbH \& Co. KgAa, Weinheim, Germany, 2005, pp. 19-354; (c) K. E. O. Ylijoki and J. M. Stryker, Chem. Rev., 2013, 113, 2244.

2 Chirality in Industry II: Developments in the Commercial Manufacture and Applications of Optically Active Compounds, ed. A. N. Collins, G. Sheldrake and J. Crosby, Wiley-VCH Verlag $\mathrm{GmbH}$, Weinheim, Germany, 1997, pp. 1-418.

3 Synthetic Applications of 1,3-Dipolar Cycloaddition Chemistry Towards Heterocycles and Natural Products, ed. A. Padwa and W. H. Pearson, Wiley, New York, 2003.

4 For reviews, see: $(a)$ C. Nájera and J. M. Sansano, Top. Heterocycl. Chem., 2008, 12, 117; (b) L. M. Stanley and M. P. Sibi, Chem. Rev., 2008, 108, 2887; (c) H. Pellisier, Tetrahedron, 2007, 63, 3235; (d) G. Pandey, P. Banerjee and S. R. Gadre, Chem. Rev., 2006, 106, 4484; (e) T. M. V. D. Pinho e Melo, Eur. J. Org. Chem., 2006, 2873; $(f)$ M. Bonin, A. Chauveau and L. Micouin, Synlett, 2006, 2349; (g) S. Husinec and V. Savic, Tetrahedron: Asymmetry, 2005, 16, 2047; (h) I. Coldham and R. Hufton, Chem. Rev., 2005, 105, 2765; (i) C. Nájera and J. M. Sansano, Angew. Chem., Int. Ed., 2005, 44, 6272.

5 P. Allway and R. Grigg, Tetrahedron Lett., 1991, 32, 5817.

6 J. M. Longmire, B. Wang and X. Zhang, J. Am. Chem. Soc., 2002, 124, 13400.

7 A. S. Gothelf, K. V. Gothelf, R. G. Hazell and K. A. Jørgensen, Angew. Chem., Int. Ed., 2002, 41, 4236.

8 J. Adrio and J. C. Carretero, Chem. Commun., 2011, 47, 6784.

9 (a) S. Watanabe, A. Tada, Y. Tokoro and S.-i. Fukuzawa, Tetrahedron Lett., 2014, 55, 1306; (b) Z.-Y. Xue, T.-L. Liu, Z. Lu, H. Huang, H.-Y. Tao and C.-J. Wang, Chem. Commun., 2010, 46, 1727.

10 (a) M. Hut'ka, T. Tsubogo and S. Kobayashi, Adv. Synth. Catal., 2013, 355, 1561; (b) K. Shimizu, K. Ogata and S.-i. Fukuzawa, Tetrahedron Lett., 2010, 51, 5068; (c) H. Y. Kim, H.-J. Shih, W. E. Knabe and K. Oh, Angew. Chem., Int. Ed., 2009, 48, 7420.
11 S. Cabrera, R. Gómez Arrayás and J. C. Carretero, J. Am. Chem. Soc., 2005, 127, 16394.

12 (a) H.-L. Teng, H. Huang, H.-Y. Tao and C.-J. Wang, Chem. Commun., $2011,47,5494$. For the use of dimethyl itaconate and 2methyleneglutarate as dipolarophiles, see: $(b)$ H.-Y. Tao, Z.-L. He, Y. Yang and C.-J. Wang, RSC Adv., 2014, 4, 16899.

13 (a) A. P. Antonchick, C. Gerding-Reimers, M. Catarinella, M. Schürmann, H. Preut, S. Ziegler, D. Rauh and H. Waldmann, Nat. Chem., 2010, 2, 735. See also: (b) R. Narayan, M. Potowski, Z.-J. Jia, A. P. Antonchick and H. Waldmann, Acc. Chem. Res., 2014, 47, 1296.

14 T.-L. Liu, Z.-Y. Xue, H.-Y. Tao and C.-J. Wang, Org. Biomol. Chem., 2011, 9, 1980.

15 (a) T. Arai, N. Yokoyama, A. Mishiro and H. Sato, Angew. Chem., Int. $E d ., 2010,49,7895$. For the obtention of exo cycloadducts, see: (b) L. M. Castelló, C. Nájera, J. M. Sansano, O. Larrañaga, A. De Cózar and F. P. Cossío, Org. Lett., 2013, 15, 2902, and references therein.

16 A. Awata and T. Arai, Chem. - Eur. J., 2012, 18, 8278.

17 T.-L. Liu, Z.-L. He, Q.-H. Li, H.-Y. Tao and C.-J. Wang, Adv. Synth. Catal., 2011, 353, 1713.

18 T.-L. Liu, Z.-L. He and C.-J. Wang, Chem. Commun., 2011, 47, 9600.

19 Q.-H. Li, T.-L. Liu, L. Wei, X. Zhou, H.-Y. Tao and C.-J. Wang, Chem. Commun., 2013, 49, 9642.

20 M. González-Esguevillas, J. Adrio and J. C. Carretero, Chem. Commun., 2013, 49, 4649.

21 Z. Wang, S. Luo, S. Zhang, W.-L. Yang, Y.-Z. Liu, H. Li, X. Luo and W.-P. Deng, Chem. - Eur. J., 2013, 19, 6739.

22 H. Takayama, Z.-J. Jia, L. Kremer, J. O. Bauer, C. Strohmann, S. Ziegler, A. P. Antonchick and H. Waldmann, Angew. Chem., Int. Ed., 2013, 52, 12404.

23 H. Clevers and R. Nusse, Cell, 2012, 149, 1192.

24 J. M. Y. Ng and T. Curran, Nat. Rev. Cancer, 2011, 11, 493.

25 Z.-Y. Xue, X. Fang and C.-J. Wang, Org. Biomol. Chem., 2011, 9, 3622.

26 T. Llamas, R. Gómez Arrayás and J. C. Carretero, Synthesis, 2007, 950.

27 Q.-H. Li, M.-C. Tong, J. Li, H.-Y. Tao and C.-J. Wang, Chem. Commun., 2011, 47, 11110.

28 Q.-H. Li, Z.-Y. Xue, H.-Y. Tao and C.-J. Wang, Tetrahedron Lett., 2012, 53, 3650 .

29 Y. Dingce, L. Quinghua and C.-J. Wang, Chin. J. Chem., 2012, 30, 2714.

30 Q. Li, C.-H. Ding, X.-H. Li, W. Weissensteiner and X.-L. Hou, Synthesis, 2012, 265.

31 (a) A. Viso, R. Fernández de la Pradilla, M. Tortosa, A. García and A. Flores, Chem. Rev., 2011, 111, PR1; (b) A. Viso, R. F. de la Pradilla, A. García, C. Guerrero-Strachan, M. Alonso, M. Tortosa, A. Flores, M. Martínez-Ripoll, I. Fonseca, I. André and A. Rodríguez, Chem. Eur. J., 2003, 9, 2867.

32 H. Xie, J. Zhu, Z. Chen, S. Li and Y. Wu, J. Org. Chem., 2010, 75, 7468.

33 Q. Hua, L. Liang, W. Xuan Chena and C.-J. Wang, Chem. Commun., 2013, 49, 6277.

34 (a) V. Nair, R. S. Menon, A. T. Biju and K. G. Abhilash, Chem. Soc. Rev., 2012, 41, 1050; (b) E. J. Corey, Angew. Chem., Int. Ed., 2002, 41, 1650; $(c)$ K. C. Nicolaou, S. A. Snyder, T. Montagnon and G. Vassilikogiannakis, Angew. Chem., Int. Ed., 2002, 41, 1668.

35 M. Potowski, M. Schürmann, H. Preut, A. P. Antonchick and H. Waldmann, Nat. Chem. Biol., 2012, 8, 428.

36 Z. He, T. Liu, H. Tao and C.-J. Wang, Org. Lett., 2012, 14, 6230.

37 K. Liu, H.-L. Teng, L. Yao, H.-Y. Tao and C.-J. Wang, Org. Lett., 2013, 15, 2253.

38 K. Dirian, M. A. Herranz, G. Katsukis, J. Malig, L. Rodríguez-Pérez, C. Romero-Nieto, V. Strauss, N. Martín and D. M. Guldi, Chem. Sci., 2013, 4, 4335.

39 (a) C. Thilgen and F. Diederich, Chem. Rev., 2006, 106, 5049; (b) Y. Wang, J. Xu, Y. Wang and H. Chen, Chem. Soc. Rev., 2013, 42, 2930.

40 (a) M. Maggini, G. Scorrano and M. Prato, J. Am. Chem. Soc., 1993, 115, 9798; (b) M. Prato and M. Maggini, Acc. Chem. Res., 1998, 31, 519.

41 S. Filippone, E. E. Maroto, A. Martín-Domenech, M. Suarez and N. Martín, Nat. Chem., 2009, 1, 578.

42 E. E. Maroto, S. Filippone, A. Martín-Domenech, M. Suarez and N. Martín, J. Am. Chem. Soc., 2012, 134, 12936.

43 E. E. Maroto, A. de Cózar, S. Filippone, A. Martín-Domenech, M. Suarez, F. P. Cossío and N. Martín, Angew. Chem., Int. Ed., 2011, 50, 6060. 
44 E. E. Maroto, M. Izquierdo, M. Murata, S. Filippone, K. Komatsu, Y. Murata and N. Martín, Chem. Commun., 2014, 50, 740.

45 K. Sawai, Y. Takano, M. Izquierdo, S. Filippone, N. Martín, Z. Slanina, N. Mizorogi, M. Waelchli, T. Tsuchiya, T. Akasaka and S. Nagase, J. Am. Chem. Soc., 2011, 133, 17746.

46 B.-C. Hong, A. K. Gupta, M.-F. Wu, J.-H. Liao and G.-H. Lee, Org. Lett., 2003, 5, 1689.

47 M. Potowski, J. O. Bauer, C. Strohmann, A. P. Antonchick and H. Waldmann, Angew. Chem., Int. Ed., 2012, 51, 9512.

48 Z.-L. He, H.-L. Teng and C.-J. Wang, Angew. Chem., Int. Ed., 2013, 52, 2934.

49 M. Potowski, A. P. Antonchick and H. Waldmann, Chem. Commun., 2013, 49, 7800.

50 For a recent review, see: V. Nair and K. G. Abhilash, Top. Heterocycl. Chem., 2008, 13, 173.

51 H. Liu, Y. Wu, Y. Zhao, Z. Li, L. Zhang, W. Yang, H. Jiang, C. Jing, H. Yu, B. Wang, Y. Xiao and H. Guo, J. Am. Chem. Soc., 2014, 136, 2625.

52 H.-L. Teng, L. Yao and C.-J. Wang, J. Am. Chem. Soc., 2014, 136, 4075.

53 (a) R. Na, C. Jing, Q. Xu, H. Jiang, X. Wu, J. Shi, J. Zhong, M. Wang, D. Benitez, E. Tkatchouk, W. A. Goddard, III, H. Guo and O. Kwon, J. Am. Chem. Soc., 2011, 133, 13337; (b) C. Jing, R. Na, B. Wang, H. Liu, L. Zhang, J. Liu, M. Wang, J. Zhong, O. Kwon and H. Guo, Adv. Synth. Catal., 2012, 354, 1023.

54 M.-C. Tong, X. Chen, H.-Y. Tao and C.-J. Wang, Angew. Chem., Int. Ed., 2013, 52, 12377.

55 H. Guo, H. Liu, F.-L. Zhu, R. Na, H. Jiang, Y. Wu, L. Zhang, Z. Li, H. Yu, B. Wang, Y. Xiao, X.-P. Hu and M. Wang, Angew. Chem., Int. Ed., 2013, 52, 12641.

56 (a) A. D. Melhado, M. Luparia and F. D. Toste, J. Am. Chem. Soc., 2007, 129, 12638; (b) M. Martín-Rodríguez, L. M. Castelló, C. Nájera, J. M. Sansano, O. Larrañaga, A. de Cózar and F. P. Cossío, Beilstein J. Org. Chem., 2013, 9, 2422; (c) J. Marco-Martínez, S. Reboredo, M. Izquierdo, V. Marcos, J. L. López, S. Filippone and N. Martín, J. Am. Chem. Soc., 2014, 136, 2897.

57 Y. Yamashita, X.-X. Guo, R. Takashita and S. Kobayashi, J. Am. Chem. Soc., 2010, 132, 3262.

58 (a) R. Robles-Machín, I. Alonso, J. Adrio and J. C. Carretero, Chem. - Eur. J., 2010, 16, 5286; (b) L. Hu and O. Ramström, Chem. Commun., 2014, 50, 3792.

59 S. Padilla, R. Tejero, J. Adrio and J. C. Carretero, Org. Lett., 2010, 12, 5608.

60 (a) C. Alemparte, G. Blay and K. A. Jørgensen, Org. Lett., 2005, 7, 4569; (b) S. Reboredo, E. Reyes, J. L. Vicario, D. Badía, L. Carrillo, A. de Cózar and F. P. Cossío, Chem. - Eur. J., 2012, 18, 7179, see also ref. 86.

61 Y. Yamashita, T. Imaizumi and S. Kobayashi, Angew. Chem., Int. Ed., 2011, 50, 4893.

62 P. Garner, L. Weerasinghe, W. Youngs, B. Wright, D. Wilson and D. Jacobs, Org. Lett., 2012, 14, 1326.

63 R. Joseph, C. Murray and P. Garner, Org. Lett., 2014, 16, 1550.

64 (a) R. Grigg and M. A. B. Sarker, Tetrahedron, 2006, 62, 10332; (b) R. Grigg, C. Kilner, M. A. B. Sarker, C. O. Cierva and H. Ali Dondas, Tetrahedron, 2008, 64, 8974.

65 T.-L. Liu, Z.-L. He, H.-Y. Tao and C.-J. Wang, Chem. - Eur. J., 2012, 18, 8042 .

66 A. D. Lim, J. A. Codelli and S. E. Reisman, Chem. Sci., 2013, 4, 650.
67 C. Chem, X. Li and S. L. Schreiber, J. Am. Chem. Soc., 2003, 125, 10174 .

68 R. Narayan, J. O. Bauer, C. Strohmann, A. P. Antonchick and H. Waldmann, Angew. Chem., Int. Ed., 2013, 52, 12404.

69 H. Ji, J. A. Gómez-Vidal, P. Martasek, L. J. Roman and R. B. Silverman, J. Med. Chem., 2006, 49, 6254.

70 M. González-Esguevillas, J. Adrio and J. C. Carretero, Chem. Commun., 2012, 48, 4649.

71 (a) O. Tsuge, S. Kanemasa, A. Hatada and K. Matsuda, Bull. Chem. Soc. Jpn., 1986, 59, 2537; (b) M. Komatsu, H. Okada, T. Akaki, Y. Oderaotoshi and S. Minakata, Org. Lett., 2002, 4, 3505.

72 J. Hernández-Toribio, S. Padilla, J. Adrio and J. C. Carretero, Angew. Chem., Int. Ed., 2012, 51, 8854.

73 A. Pascual-Escudero, M. González-Esguevillas, S. Padilla, J. Adrio and J. C. Carretero, Org. Lett., 2014, 16, 2228.

74 W. S. Jen, J. J. M. Wiener and D. W. C. MacMillan, J. Am. Chem. Soc., 2000, 122, 9874.

75 J. L. Vicario, S. Reboredo, D. Badia and L. Carrillo, Angew. Chem., Int. Ed., 2007, 46, 5168.

76 M.-X. Xue, X.-M. Zhang and L.-Z. Gong, Synlett, 2008, 691.

77 Y.-K. Liu, H. Liu, W. Du, L. Yue and Y.-C. Chen, Chem. - Eur. J., 2008, 14, 9873.

78 (a) X.-H. Chen, W.-Q. Zhang and L.-Z. Gong, J. Am. Chem. Soc., 2008, 130, 5652; (b) J. Yu, F. Shi and L.-Z. Gong, Acc. Chem. Res., 2011, 44, 1156.

79 S. Reboredo, J. L. Vicario, D. Badía, L. Carrillo and E. Reyes, Adv. Synth. Catal., 2011, 353, 3307.

80 S. Lin, L. Deiana, G.-L. Zhao, J. Sun and A. Córdova, Angew. Chem., Int. Ed., 2011, 50, 7624.

81 N. Fernández, L. Carrillo, J. L. Vicario, D. Badía and E. Reyes, Chem. Commun., 2011, 47, 12313.

82 A. Fraile, D. M. S. Schietroma, A. Albrecht, R. L. Davis and K. A. Jørgensen, Chem. - Eur. J., 2012, 18, 2773.

83 J. Yu, L. He, X.-H. Chen, J. Song, W.-J. Chen and L.-Z. Gong, Org. Lett., 2009, 11, 4946.

84 J. Yu, W.-J. Chen and L.-Z. Gong, Org. Lett., 2010, 12, 4050.

85 C. Wang, X.-H. Chen, S.-M. Zhou and L.-Z. Gong, Chem. Commun., 2010, 46, 1275.

86 X.-H. Chen, Q. Wei, S.-W. Luo, H. Xiao and L.-Z. Gong, J. Am. Chem. Soc., 2009, 131, 13819.

87 F. Shi, S.-W. Luo, Z.-L. Tao, L. He, J. Yu, S.-J. Tu and L.-Z. Gong, Org. Lett., 2011, 13, 4680.

88 W.-J. Liu, X.-H. Chen and L.-Z. Gong, Org. Lett., 2008, 10, 5357.

89 F. Shi, R.-Y. Zhu, W. Dai, C.-S. Wang and S.-J. Tu, Chem. - Eur. J., 2014, 20, 2597.

90 F. Shi, Z.-L. Tao, S.-W. Luo, S.-J. Tu and L.-Z. Gong, Chem. - Eur. J., 2012, 18, 6885.

91 F. Shi, R.-Y. Zhu, X. Liang and S.-J. Tu, Adv. Synth. Catal., 2013, $355,2447$.

92 C. Guo, J. Song and L.-Z. Gong, Org. Lett., 2013, 15, 2676.

93 J.-F. Bai, L.-L. Wang, L. Peng, Y.-L. Guo, J.-N. Ming, F.-Y. Wang, X.-Y. Xu and L.-X. Wang, Eur. J. Org. Chem., 2011, 4472.

94 L. Tian, G.-Q. Xu, Y.-H. Li, Y.-M. Liang and P.-F. Xu, Chem. Commun., $2014,50,2428$.

95 L. Wang, X.-M. Shi, W.-P. Dong, L.-P. Zhu and R. Wang, Chem. Commun., 2013, 49, 3458. 